\title{
Robust stability analysis of a dc/dc buck converter under multiple parametric uncertainties
}

\author{
Sharmila Sumsurooah \\ The Department of Electrical and Electronic Engineering \\ The University of Nottingham \\ Nottingham NG7 2RD, UK \\ sharmila.sumsurooah@nottingham.ac.uk \\ Milijana Odavic, Member, IEEE, \\ The Department of Electronic and Electrical Engineering \\ The University of Sheffield \\ Sheffield S10 2TN, UK \\ M.Odavic@sheffield.ac.uk \\ Serhiy Bozhko, Member, IEEE, \\ The Department of Electrical and Electronic Engineering \\ The University of Nottingham \\ Nottingham NG7 2RD, UK \\ Serhiy.Bozhko@nottingham.ac.uk \\ Dushan Boroyevich, Fellow, IEEE \\ Centre for Power Electronics Systems \\ Virginia Polytechnic Institute and State University \\ Blacksburg VA 24061-0111, USA \\ dushan@vt.edu
}

\begin{abstract}
Stability studies are a crucial part of the design of power electronic systems, especially for safety critical applications. Standard methods can guarantee stability under nominal conditions but do not take into account the multiple uncertainties that are inherent in the physical system or in the system model. These uncertainties, if unaccounted for, may lead to highly optimistic or even erroneous stability margins. The structured singular value-based $\mu$ method justifiably takes into account all possible uncertainties in the system. However, the application of the $\mu$ method to power electronic systems with multiple uncertainties is not widely discussed in the literature. This work presents practical approaches to applying the $\mu$ method in the robust stability analysis of such uncertain systems. Further, it reveals the significant impact of various types of parametric uncertainties on the reliability of stability assessments of power electronic systems. This is achieved by examining the robust stability margin of the dc/dc buck converter system, when it is subject to variations in system load, line resistance, operating temperature and uncertainties in the system model. The $\mu$ predictions are supported by time domain simulation and experimental results.
\end{abstract}

Index Terms-Robust stability analysis, dc/dc buck power electronic converter, Linear fractional transformation, Structured singular value, $\mu$ analysis.

\section{INTRODUCTION}

$\mathbf{T}$ RANSPORT accounts for nearly two thirds of the global crude oil consumption and about a quarter of carbon dioxide emissions [1]. In order to address the issues of energy security and greenhouse emissions, a shift towards more electric-intensive architectures of transportation system such as land vehicles, aviation and ships is not only needed but seems inevitable. This implies a partial to complete electrification of transport modes, which are referred to as "more electric" transport (MET) [2]. Power electronics (PE) lies at the heart of this technology transition. Yet, the susceptibility of power electronic systems to instability remains an important issue that needs attention [3], [4], [5], [6]. Finding an answer to this problem is crucial, particularly for safety critical applications. System stability may be assessed at both the small and large signal level. Lyapunov theory of stability is generally employed to estimate large signal stability regions of the system [7]. This work focusses on small-signal stability analysis, which is one of the important concerns in the reliable operation of the system [4], [5], [8].

While power electronic technology is fast evolving, the methods that are widely employed to assess the small-signal system stability are mostly based on classical techniques. These include the eigenvalue approach and impedance methods based on Nyquist stability criterion [9], [10]. Since classical methods work on the nominal model of the physical system, the outcome of the stability assessment is heavily dependent on the quality of the system model [3], [11]. The model may be refined to great detail by matching its response to that of the physical system. Yet, in practice, excessive model refinement is unlikely to be viable or practical. Further, the exact values of system components may not be known accu- 
rately. For instance, system parasitics, often hard to quantify, can have a significant influence on the quality of the model. The power supply and external filters, to be connected on site, may be unknown at the design stage. This may significantly alter the impedance of the power stage. Hence, the nominal system is bound to contain model uncertainties. From another perspective, even though a nominal model is deemed to be accurate, it may not truly represent the actual system, which is generally subject to various operating conditions uncertainties. Electrical power systems may be exposed to large variations in their loads. In aerospace applications power electronic systems may be exposed to temperatures typically ranging from -40 ${ }^{\circ} C$ to $125^{\circ} C$ [12]. In practice, PE systems may be subject to the aforementioned types of uncertainties that are normally not accounted for by classical techniques. In order to keep pace with evolving technology in power electronics, there is a need to adopt new analysis techniques that duly incorporate uncertainties inherently present in the system. The structured singular value (SSV) based $\mu$ method is a robust stability analysis tool that justifiably takes into account all possible system perturbations. This work focuses on parametric uncertainties [13], [14]. Recent works [15] and [16] demonstrate the effect of parametric uncertainties on the stability of PE-based systems.

Classical methods, that are designed for single-input-singleoutput systems, can include multiple uncertainties in stability analysis. For instance, the authors in [17] have incorporated uncertainties in the application of the classical impedancebased energy source analysis consortium method. However, the process involves extensive parameter iteration and system linearisation. In addition, the eigenvalue method is applied in combination with probabilistic stability method such as the Monte Carlo simulation [18], [19]. However, when such classical methods are applied to multi-input multi-output (MIMO) systems, there is no guarantee that all possible combinations of uncertainties acting on the system can be evaluated to identify the most critical scenario with respect to stability [18], [20], [21]. The results may not always be reliable. Conversely, the $\mu$ method is a robust, reliable and deterministic approach that can be employed to incorporate uncertainties in stability analysis of MIMO systems [14], [22], [23], [24]. Further, it does not involve a laborious iterative process. In contrast with classical techniques based on Nyquist stability criterion, the $\mu$ method provides a direct and explicit measure of the robust stability margin as a percentage of the maximum change that is allowed in an uncertain parameter for the system to remain stable. Despite the aforementioned advantages, a major drawback of the $\mu$ method is that $\mu$ cannot be computed as an exact value particularly for large size problems, since the computational burden increases exponentially with the size of the problem. A lower bound and an upper bound are calculated instead of its exact value. It has been reported in the literature that the method can be computationally expensive for the analysis of complex systems with a large number of uncertainties [25], [26]. However, a number of algorithms has been developed to reduce the gap between the $\mu$ bounds while maintaining reasonable computational time [25], [27], [28], [29]. Tools to optimise state space system models and model reduction methods may be employed to decrease the computational burden [30].

Although the strength of the $\mu$ tool lies in the fact that it can be applied to system models with multiple uncertainties, the benefits of this powerful feature have been utilised or examined in a limited number of studies [21], [31]. This may be due to a few limitations that tend to make the approach hard to apply. First is the complexity of the underlying $\mu$ theory. Although a great amount of literature is devoted to the theoretical framework, certain key aspects of the $\mu$ method are not examined from an engineering viewpoint, particularly when treating multiple uncertainties [13], [32]. Further, the methodology required to practically apply the $\mu$ tool to $\mathrm{PE}$ systems subject to single and multiple uncertainties is not widely discussed in the literature. In addition, the $\mu$ method is generally applied to linear systems while most systems analysed have nonlinear behaviour. A few works in the literature have successfully applied the $\mu$ method to analyse stability of conventional power systems [33], [34], [35], [36], [37], [38]. However, the methodology applied through associated software is not discussed and multiple uncertainties are not considered. Certain works such as [25], [39] have treated the practical aspects of the $\mu$ approach in good depth. While the results are clearly examined, the method employed are not presented in a manner that is comprehensive enough to reapply them to other systems. Other works related to the $\mu$ approach deal with $\mu$ sensitivity or the design of a robust controller as opposed to the robust stability of PE systems [18], [40], [41], [42], [43].

In order to make the $\mu$ approach more application-friendly and to fully realise the benefits of the method, the current work addresses the aforementioned limitations of the $\mu$ tool. First, it presents practical approaches to apply the $\mu$ tool to a PE system subject to single and multiple uncertainties. In [44], the methodology to apply the $\mu$ tool to determine the robust stability margin of a dc/dc buck converter subject to uncertainty in load and operating temperature is presented. This work extends the practical application of the $\mu$ tool to assess the robust stability of the aforementioned $\mathrm{dc} / \mathrm{dc}$ buck converter system when it is subject to multiple uncertainties in the nominal system model. It shows how model uncertainties, which may be known to different level of accuracy, can be incorporated in robust stability analysis of the system, without compromising the reliability of the results. For the purpose of demonstrating how the $\mu$ tool supports a possible trade-off between accuracy and simplicity in the system model, this work evaluates the robust stability of the experimentally-refined model of the system under study against that of its approximate system model. As discussed earlier, this is particularly relevant to the design engineer who, in practice, may not have sufficient information of the exact values of all system components. Secondly, this work applies the modelling methodology, developed and presented in [45], [46], to convert the nonlinear model of the dc/dc buck converter under study into an equivalent linear model that is suited for $\mu$ analysis. The modelling method is based on the symbolic linearisation about an arbitrary operating point, and also caters for dependences of operating points on parameter 
variations. In contrast, the work in [21] does not take into account dependences of operating points on uncertainties in system parameters. In addition, this work demonstrates the significance of the robust stability measure $\mu$ for a practical PE system subject to multiple parametric uncertainties. In [47], the authors demonstrate the usefulness of $\mu$ by presenting the concept of the hypercube. It has been demonstrated that a system subject to $N$ parametric uncertainties is guaranteed robustly stable within a hypercube of dimension $N$ and of coordinate size $1 / \mu$, centred about a nominal point [25], [47]. This work applies the hypercube concept to demonstrate the significance of the $\mu$ results for the practical dc/dc buck converter system under investigation when it is subject to both single and multiple parametric uncertainties. The paper also gauges the impact of uncertainties on the reliability of stability assessments, and consequently call for the adoption of robust tools in the stability analysis of PE systems for MET applications. Experimental validation of the $\mu$ results is provided for the buck converter system under a single parametric uncertainty and then when it is exposed to an additional parametric uncertainty. Time domain simulations are used to evaluate the $\mu$ predictions for the analysed system when it is subject to multiple parametric uncertainties.

\section{THEORETICAL FRAMEWORK}

A great advantage of the $\mu$ approach is that it works with the uncertain system model as opposed to the nominal model [14], [22], [23], [24]. An uncertain system model considers not only nominal values of the system parameters but also the possible range of parameter changes. In order to analyse stability of uncertain systems, the principle of structured singular value ( $\mu$ ) can be used. Prior to applying $\mu$ analysis, the system must be expressed in the linear fractional transformation (LFT) form [13], [23]. The aim is to verify that a system remains stable for all conditions that may arise within the defined uncertainty set, in which case it is said that the system is robustly stable.

LFT is a modelling technique which is employed to "pull out" the indeterminate part from the known part of a system model and place it in the feedback form. If a general uncertain parameter $P$ is considered to be bounded in the region $\left[P_{\min }\right.$, $\left.P_{\max }\right]$, it may be represented in its normalised form $\delta_{P}$ bounded within $[-1,1]$. The parameter $P$ can be modelled as an LFT in $\delta_{P}$ in the expression (1) and in the matrix form in Fig. 1a [14], [48], where the input and output signals of the parameter are denoted as $u_{s p}$ and $y_{s p}$ respectively, while $u_{\Delta p}$ and $y_{\Delta p}$ denote the output and input of $\delta_{P}$ [14], [39].

$$
\begin{aligned}
P & =P_{o}+P_{o} P_{v a r} \delta_{P}, \quad \delta_{P} \in[-1,1] \\
\text { where } P_{o} & =\left(P_{\min }+P_{\max }\right) / 2 \\
\text { and } \quad P_{\text {var }} & =\frac{\left(P_{\max }-P_{\min }\right)}{2} / \frac{\left(P_{\max }+P_{\min }\right)}{2}
\end{aligned}
$$

The model of an entire system with parametric uncertainties can also be represented in the LFT form as shown in Fig. 1b and in the general LFT equation in (2) [23], [49]. The diagonal uncertainty matrix $\Delta$ as shown in Fig. 1b contains the set of uncertainties in their normalised form, that have been extracted from the uncertain system model.

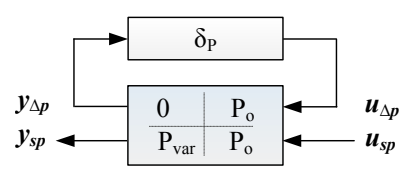

(a)

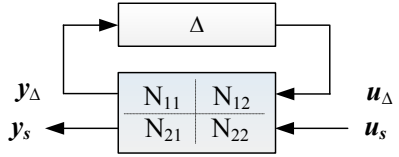

(b)
Fig. 1: (a) Uncertain parameter $P$ as an LFT (b) General uncertain system model $N \Delta$ as an LFT

$$
F_{u}(N, \Delta)=\frac{y_{s}}{u_{s}}=N_{22}+N_{21} \Delta\left(I-N_{11} \Delta\right)^{-1} N_{12}
$$

Referring to the general LFT expression (2), it can be seen that the only source that can cause the system $N \Delta$ to become unstable is the feedback term $(I-M \Delta)^{-1}$ where $M=N_{11}$ [23]. The stability of the whole system therefore rests on the stability of the subsystem $(I-M \Delta)^{-1}$. The robust stability condition for structured uncertainties is given by the structured singular value (SSV), as defined in (3).

$$
\mu_{\Delta}(M)=\frac{1}{\min [\bar{\sigma}(\Delta): \operatorname{det}(I-M \Delta)=0, \Delta \text { structured }]}
$$

The structured singular value, commonly denoted as $\mu$, identifies the smallest perturbation matrix $(\Delta)$ that destabilises the system. Thus, the SSV theory gives necessary and sufficient conditions for stability robustness [13]. For the identified smallest perturbation matrix $(\Delta)$, the system poles are at the imaginary axis [14], [22]. If $\mu$ is less than 1 , the system is guaranteed to be stable for the entire uncertainty set. Further, as metioned earlier in this work, for a system subject to $N$ parametric uncertainties, $\mu$ provides the largest hypercube of dimension $N$, centred about the nominal point and of coordinate size $1 / \mu$, within which the system can be guaranteed robustly stable. $1 / \mu$ is a measure of the robust stability margin of the system [25], [45].

Since, it is generally hard to compute the exact value of $\mu$, a lower and an upper bound to $\mu$ are calculated. The concept of the $\mu$ bounds is illustrated in Fig. 2. A lower bound $\mu$ (which is also the upper bound of the robust stability margin) provides a sufficient condition of the instability of the system for the model perturbation $\Delta=1 / \mu$ [25]. On the other hand, the upper bound $\bar{\mu}$ (which is also the lower bound of the robust stability margin) provides a sufficient condition that guarantees stability of the system for all perturbations that are smaller than $1 / \bar{\mu}[25]$.

Although, $\mu$ is not guaranteed to be equal to $\mu$, it is always computed at the boundary of stability [28]. Further, $\mu$ yields the worse case perturbation model for a system [28]. On the other hand, the upper bound $\bar{\mu}$ is generally higher than $\mu$ and tends to be conservative. The worse ratio of $\mu / \bar{\mu}$ has been reported to be equal to 0.85 while in most cases the ratio is close to unity [14]. The lower bound $\mu$ is therefore generally 


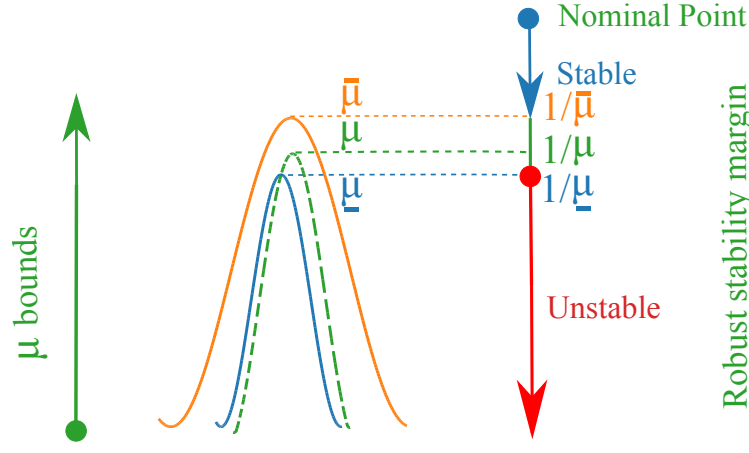

Fig. 2: Representative figure to illustrate $\mu$ bounds and robust stability margin

close to $\mu$ [29]. For the aforementioned reasons, the lower bound $\mu$ is used as a good estimate of $\mu$ in this study.

\section{SySTEM MODELLING FOR $\mu$ ANALYSIS}

The electrical network of the MET will consist of a large number of power electronic converters. A typical subdistribution system in the MET architecture can be represented by a source feeding power through a distribution line and an input filter to a power electronic interfaced load. The buck converter system, as shown in Fig. 3, has been chosen as a representation of the aforementioned sub-distribution system. The general modelling method, proposed in [45], [46], is used in this work to convert the nonlinear buck converter system model into an equivalent linear model, which is valid for $\mu$ analysis over a range of operating points and parameter variations. The method is based on symbolic linearisation around an arbitrary equilibrium point. All elements in the system model are explicitly expressed in terms of definable system parameters and input only, and for instance not in terms of indeterminate equilibrium states. The method, not being the focus in this study, is not emphasised in this work. The reader is referred to [45], [46] for more details. Further, in this work, a modular modelling approach is used in that the small-signal ac models of the power stage and the controller are built separately and then combined to yield the complete linear time invariant model of the closed loop converter, as is presented in the rest of this section [40], [50], [51], [52].

\section{A. The experimental buck converter}

The experimental closed loop buck converter, that is used in this study, is depicted in the circuit model in Fig. 3. The power stage is made up of an inductor-capacitor (LC) input filter and the open loop buck converter [50]. The U3825 PWM controller consists of a Type III analogue compensator and a modulator [53]. The sawtooth generator of the modulator generates a sawtooth waveform of peak voltage $\left(V_{p p}\right)$ measured as $3.52 \mathrm{~V}$. The modulation gain $f_{m}$ is given by $1 / V_{p p}$ and is equal to 0.284 [54]. The switching frequency has been measured as $51.2 \mathrm{kHz}$. The small-signal ac model of the closed loop controlled buck converter, which is illustrated in Fig. 3, is developed in the subsequent subsections. The system parameters are defined as "initial" parameters in Table I.

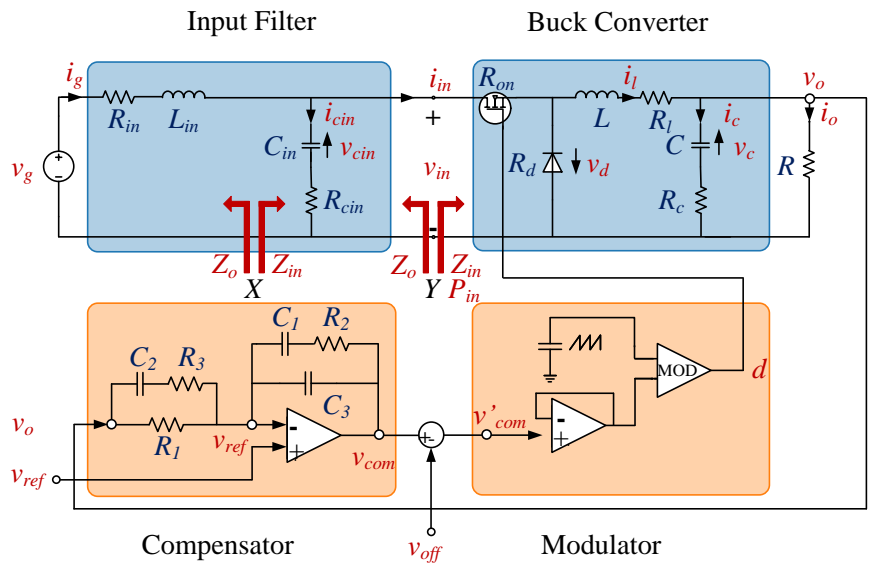

Fig. 3: Circuit representation of the closed loop controlled buck converter with input filter

\section{B. Power stage model}

The buck converter has two operating modes over one switching period. Equations (4) and (5) describe the dynamics of the power stage when the switch is on and off respectively. The parameters in (4) - (5) are shown in Fig. 3 and described in Table I.

On-state period :

$\frac{d i_{g}}{d t}=\left(v_{g}-i_{g} R_{i n}-v_{c i n}-\left(i_{g}-i_{l}\right) R_{c i n}\right) / L_{i n}$

$\frac{d v_{c i n}}{d t}=\left(i_{g}-i_{l}\right) / C_{i n}$

$\frac{d i_{l}}{d t}=\left(v_{c i n}+\left(i_{g}-i_{l}\right) R_{c i n}-\left(v_{o}+i_{l} R_{l o n}\right)\right) / L$

$\frac{d v_{c}}{d t}=\left(i_{l}-v_{o} / R\right) / C$

$v_{o}(t)=\left(R_{c} i_{l}+v_{c}\right)\left(R /\left(R_{c}+R\right)\right)$

Off-state period:

$\frac{d i_{g}}{d t}=\left(v_{g}-i_{g} R_{i n}-v_{c i n}-i_{g} R_{c i n}\right) / L_{i n}$

$\frac{d v_{c i n}}{d t}=i_{g} / C_{i n}$

$\frac{d i_{l}}{d t}=\left(-v_{d}-v_{o}-i_{l} R_{l d}\right) / L$

$\frac{d v_{c}}{d t}=\left(i_{l}-v_{o} / R\right) / C$

$v_{o}(t)=\left(R_{c} i_{l}+v_{c}\right)\left(R /\left(R_{c}+R\right)\right)$

where $R_{\text {lon }}=R_{l}+R_{\text {on }}, \quad R_{l d}=R_{l}+R_{d}$,

The averaging modelling method is applied. Equations (4) and (5) are firstly represented as the state equations (6) and (7) respectively, with state vectors $x(t): \quad\left[i_{g}(t), v_{c i n}(t), i_{l}(t), v_{c}(t)\right]$, input vector 
$u(t):\left[v_{g}(t), v_{d}(t)\right]$ and output vector $y(t):\left[v_{o}(t)\right]$.

$$
\begin{array}{ll}
\frac{d x(t)}{d t}=A_{1} x(t)+B_{1} u(t), & y(t)=E_{1} x(t)+F_{1} u(t) \\
\frac{d x(t)}{d t}=A_{2} x(t)+B_{2} u(t), & y(t)=E_{2} x(t)+F_{2} u(t)
\end{array}
$$

Then, averaging (6) and (7) over a switching period produces the system model as given by (8). This is based on the duty cycle $d(t)$ during the on-state period and $d^{\prime}(t)=1-d(t)$ during the off-state period.

$$
\begin{aligned}
\frac{d \overline{x(t)}}{d t} & =\left[d(t) A_{1}+d^{\prime}(t) A_{2}\right] \overline{x(t)}+\left[d(t) B_{1}+d^{\prime}(t) B_{2}\right] \overline{u(t)} \\
\frac{y(t)}{y(t)} & =\left[d(t) E_{1}+d^{\prime}(t) E_{2}\right] \overline{x(t)}+\left[d(t) F_{1}+d^{\prime}(t) F_{2}\right] \overline{u(t)}
\end{aligned}
$$

The averaged model (8) is nonlinear as it involves the multiplication of time varying quantities. In order to obtain the linear small-signal ac model of the system, the averaged model must be linearised about a dc steady state operating point. To that end, the variables in (8) are firstly expanded in terms of their $\mathrm{dc}$ and ac components. The averaged state vector $\bar{x}(t)$, input vector $\bar{u}(t)$, output vector $\bar{y}(t)$ and duty cycle $d(t)$ are expressed in terms of their dc steady state values $X, U, Y$ and $D$ with superimposed small ac variations $\hat{x}(t), \hat{u}(t), \hat{y}(t)$ and $\hat{d}(t)$ respectively as shown in (9). It can be shown that $\hat{d}(t)=-\hat{d}^{\prime}(t)$.

$$
\begin{array}{ll}
\overline{x(t)}=X+\hat{x}(t), & \overline{u(t)}=U+\hat{u}(t) \\
\overline{y(t)}=Y+\hat{y}(t), & d(t)=D+\hat{d}(t)
\end{array}
$$

After substituting (9) in (8) and collecting common terms, the averaged state space model can be written as (10) and (11).

$$
\begin{aligned}
& \frac{d(\hat{x}(t))}{d t}=A X+B U \\
& +A \hat{x}(t)+B \hat{u}(t)+\left(\left(A_{1}-A_{2}\right) X+\left(B_{1}-B_{2}\right) U\right) \hat{d}(t) \\
& +\left(A_{1}-A_{2}\right) \hat{x}(t) \hat{d}(t)+\left(B_{1}-B_{2}\right) \hat{u}(t) \hat{d}(t) \\
& Y+\hat{y}(t)=E X+F U \\
& +E \hat{x}(t)+F \hat{u}(t)+\left(\left(E_{1}-E_{2}\right) X+\left(F_{1}-F_{2}\right) U\right) \hat{d}(t) \\
& +\left(E_{1}-E_{2}\right) \hat{x}(t) \hat{d}(t)+\left(F_{1}-F_{2}\right) \hat{u}(t) \hat{d}(t)
\end{aligned}
$$

The averaged model in steady state corresponds to the dc terms in (10) and (11) and is given as (12).

$$
0=A X+B U, \quad Y=E X+F U
$$

where $A=A_{1} D+A_{2} D^{\prime}, \quad B=B_{1} D+B_{2} D^{\prime}$

$$
\begin{aligned}
E & =E_{1} D+E_{2} D^{\prime}, \quad F=F_{1} D+F_{2} D^{\prime} \\
D^{\prime} & =1-D
\end{aligned}
$$

The equilibrium state can be computed as $\left(X=-B U A^{-1}\right)$ with $U=\left[V_{g}, V_{d}\right]^{T}$ based on (12).

Symbolic linearisation of the averaged model about the quiescent dc point, given by (12), involves neglecting the second order nonlinear terms in (10) - (11) as they are very small in magnitude when compared to the linear terms. The resulting linearised small-signal ac model, in symbolic form, is obtained as (13) - (14) in its full form.

$$
\begin{aligned}
\frac{d \hat{i}_{g}(t)}{d t} & =\frac{-\left(R_{c i n}+R_{i n}\right)}{L_{i n}} \hat{i}_{g}(t)-\frac{1}{L_{i n}} \hat{v}_{c i n}(t) \\
& +\frac{D R_{c i n}}{L_{i n}} \hat{i}_{l}(t)+\frac{1}{L_{i n}} \hat{v}_{g}(t)+p_{1} \hat{d}(t) \\
\frac{d \hat{v}_{c i n}(t)}{d t} & =\frac{1}{C_{i n}} \hat{i}_{g}(t)-\frac{D}{C_{i n}} \hat{i}_{l}(t)+p_{2} \hat{d}(t) \\
\frac{d \hat{i}_{l}(t)}{d t} & =\frac{D R_{c i n}}{L} \hat{i}_{g}(t)+\frac{D}{L} \hat{v}_{c i n}(t)+\frac{q}{L} \hat{i}_{l}(t) \\
& -\frac{R}{L\left(R+R_{c}\right)} \hat{v}_{c}(t)+\frac{D-1}{L} \hat{v}_{d}(t)+p_{3} \hat{d}(t) \\
\frac{d \hat{v}_{c}(t)}{d t} & =\frac{R}{C\left(R+R_{c}\right)} \hat{i}_{l}(t)-\frac{1}{C\left(R+R_{c}\right)} \hat{v}_{c}(t) \\
\hat{v}_{o}(t) & =\frac{R_{c} R}{R_{c}+R} \hat{i}_{l}(t)+\frac{R}{R_{c}+R} \hat{v}_{c}(t)
\end{aligned}
$$

where $k_{1}=R+R_{l}+R_{d}+D^{2}\left(R_{i n}-R_{\text {cin }}\right)$

$$
\begin{aligned}
& +D\left(R_{\text {cin }}-R_{d}+R_{o n}\right) \\
k_{2} & =\frac{R R_{c}}{R+R c}, \quad k_{3}=D V_{g}+(D-1) V_{d} \\
k_{4} & =\left(R+R_{l}+R_{d}\right) V_{g}+D R_{i n} V_{d}+D R_{c i n} V_{g} \\
& +D\left(R_{o n}-R_{d}\right) V_{g}-D^{2}\left(R_{c i n} V_{g}+R_{\text {in }} V_{d}\right) \\
p_{1} & =\frac{R_{\text {cin }} k_{3}}{L_{i n} k_{1}} \\
p_{2} & =-\frac{k_{3}}{C_{\text {in }} k_{1}} \\
p_{3} & =\frac{k_{3}\left(R_{d}-R_{\text {cin }}-R_{o n}\right)}{L k_{1}}+\frac{k_{4}}{L k_{1}}+\frac{D R_{\text {cin }} k_{3}}{L k_{1}}+\frac{V_{d}}{L}
\end{aligned}
$$

\section{Controller model}

The transfer functions of the compensator and modulator, shown in Fig. 3, can be written as (15) and (16) respectively.

$$
\begin{aligned}
G_{c}(s) & =\frac{v_{c o m}(s)-v_{r e f}(s)}{v_{r e f}-v_{o}(s)} \\
& =\frac{k\left(s+w_{z 1}\right)\left(s+w_{z 2}\right)}{s\left(s+w_{p 2}\right)\left(s+w_{p 3}\right)} \\
d(s) & =f_{m}\left(v_{c o m}(s)-v_{o f f}(s)\right)
\end{aligned}
$$

$$
\begin{aligned}
\text { where } k & =\frac{\left(R_{1}+R_{3}\right)}{R_{1} R_{3} C_{3}} \\
w_{z 1} & =\frac{1}{C_{1} R_{2}}, \quad w_{z 2}=\frac{1}{C_{2}\left(R_{1}+R_{3}\right)} \\
w_{p 2} & =\frac{1}{C_{2} R_{3}}, \quad w_{p 3}=\frac{\left(C_{1}+C_{3}\right)}{C_{1} C_{3} R_{2}}
\end{aligned}
$$

Based on the above transfer functions, the state equations of the small-signal ac model of the controller can be obtained as (17) and (18) respectively with state vector 
$\hat{x}(t):\left[\hat{x}_{5}(t), \hat{x}_{6}(t), \hat{x}_{7}(t)\right]$, input vector $\hat{u}(t):\left[\hat{v}_{r e f}(t), \hat{v}_{o f f}(t)\right]$ and output vector $\hat{y}(t):[\hat{d}(t)]$.

$$
\begin{aligned}
\dot{\hat{x}}_{5}(t) & =-\left(w_{p 2}+w_{p 3}\right) \hat{x}_{5}(t)-w_{p 2} w_{p 3} \hat{x}_{6}(t) \\
& +\hat{v}_{r e f}(t)-\hat{v}_{o}(t) \\
\dot{\hat{x}}_{6}(t) & =\hat{x}_{5}(t) \\
\dot{\hat{x}}_{7}(t) & =\hat{x}_{6}(t) \\
\hat{d}(t) & =m_{1} \hat{x}_{5}(t)+m_{2} \hat{x}_{6}(t)+m_{3} \hat{x}_{7}(t) \\
& \left.+f_{m} \hat{v}_{r e f}(t)\right)-f_{m} \hat{v}_{o f f}(t)
\end{aligned}
$$

where $m_{1}=f_{m} k, \quad m_{2}=f_{m} k\left(w_{z 1}+w_{z 2}\right)$, $m_{3}=f_{m} k w_{z 1} w_{z 2}$

\section{Closed loop controlled converter model}

At this point, the power stage and controller models, derived in the earlier sections, can be combined to yield the small-signal ac model of the closed loop controlled buck converter. The process involves replacing $\hat{v}_{o}(t)$ in (17) with expression (14), and $\hat{d}(t)$ in (13) with expression (18). The resulting state space model has state vector $\hat{x}(t)=\left[\hat{i}_{g}(t), \hat{v}_{c i n}(t), \hat{i}_{l}(t), \hat{v}_{c}(t), \hat{x}_{5}(t), \hat{x}_{6}(t), \hat{x}_{7}(t)\right]$, input vector $\hat{u}(t)=\left[\hat{v}_{g}(t), \hat{v}_{d}(t), \hat{v}_{r e f}(t), \hat{v}_{o f f}(t)\right]$ and output vector $\hat{y}(t)=\hat{v}_{o}(t)$, and is shown in (20), in the form $\left(\begin{array}{c}A \\ E F\end{array}\right)$.

All elements in the system model (20) must be expressed as functions of definable system parameters and inputs only. Hence, the duty cycle $D$ in (20) is expressed in terms of determinate elements by solving the quadratic equation (19) which is based on the equivalent steady-state model of the buck converter in Fig.4. This modelling step serves to cater for system nonlinearities in the system model (20).

$$
\begin{aligned}
D^{2} V_{o} \frac{R_{i n}}{R} & +D\left[-V_{g}-V_{d}+V_{o} \frac{\left(R_{o n}-R_{d}\right)}{R}\right] \\
& +\left[V_{d}+V_{o} \frac{\left(R+R_{d}+R_{l}\right)}{R}\right]=0
\end{aligned}
$$

Further, all elements in the developed model must be in their rational forms in order to allow conversion of the system model to its corresponding LFT configuration. The duty cycle $D$ in (13), which is obtained as a solution to (19), is irrational and is therefore approximated by a polynomial expansion. Fig. 5 shows the rational zeroth, first and second order Taylor series expansions of duty cycle D about the nominal operating point. Of note is that the nominal resistive load is $2.5 \Omega$ as given in Table I, and the zeroth order Taylor approximation of the duty cycle is 0.2768 . Although the second order Taylor series provides the best approximation, as can be seen in Fig. 5, the first order approximation is used in this section of the work, not to unnecessary increase the computational complexity.

It is to be added that all the elements of the developed model are rational, in symbolic form and expressed in terms of system parameters and system inputs only. The system model (20), referred to as the linear equivalent model of the buck converter, is suited for $\mu$ analysis over a range of operating points and parameter variations [45], [46].

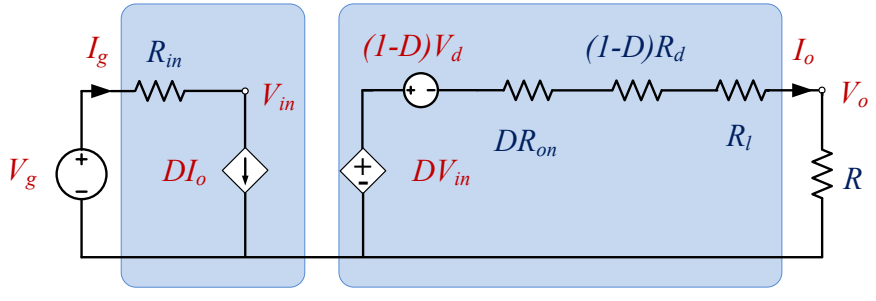

Fig. 4: Model of the buck converter in steady state

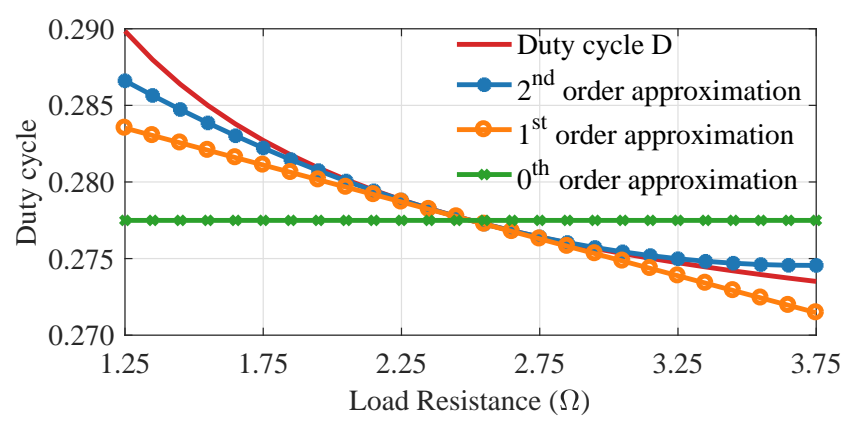

Fig. 5: Duty cycle $D$ as a function of load $R$

\section{SYSTEM MODEL REFINEMENT OF THE EXPERIMENTAL PROTOTYPE}

The system model of the experimental buck converter shown in Fig. 3 has been built in Simulink ${ }^{\circledR}$ environment. One of the initial objectives of the study was to obtain the refined system model of the experimental prototype to predict the behaviour of the system such as borderline stability with fairly good accuracy. The procedure for refining the system model is done in three main stages, as described in this section.

\section{A. Initial system model}

The first step consists in defining the initial average model of the buck converter under study based on available data and nameplate information, as defined under "the initial parameter values" in Table I. Many of these parameter values can be further refined to increase model fidelity.

\section{B. Individual system components models}

The second step of the process models individual system components through experimental measurements. It has been shown that non-idealities such as parasitic resistances in wiring and power supply, the equivalent series resistance (ESR) of the capacitors and inductors as well as, the voltage drop in the diode and the on-resistance of the switch transistor have significant impact on the accuracy of the model. The experimental measurements of a few system components are described below.

The output impedance of the power supply has been measured when connected to different loads and set to different voltages, as shown in Fig. 6. A network analyser has been employed to obtain the dynamic measurements [50]. The best estimate of the experimental measurements of the power supply impedance, through curve fitting, is found to 


$\left[\begin{array}{ccccccc|cccc}\frac{-\left(R_{\text {cin }}+R_{i n}\right)}{L_{i n}} & -\frac{1}{L_{i n}} & \frac{D R_{\text {cin }}}{L_{i n}} & 0 & p_{1} m_{1} & p_{1} m_{2} & p_{1} m_{3} & \frac{1}{L_{i n}} & 0 & p_{1} f_{m} & -p_{1} f_{m} \\ \frac{1}{C_{i n}} & 0 & -\frac{D}{C_{i n}} & 0 & p_{2} m_{1} & p_{2} m_{2} & p_{2} m_{3} & 0 & 0 & p_{2} f_{m} & -p_{2} f_{m} \\ \frac{D R_{c i n}}{L} & \frac{D}{L} & \frac{q}{L} & -\frac{R}{L\left(R+R_{c}\right)} & p_{3} m_{1} & p_{3} m_{2} & p_{3} m_{3} & 0 & \frac{D-1}{L} & p_{3} f_{m} & -p_{3} f_{m} \\ 0 & 0 & \frac{R}{C\left(R+R_{c}\right)} & -\frac{1}{C\left(R+R_{c}\right)} & 0 & 0 & 0 & 0 & 0 & 0 & 0 \\ 0 & 0 & -\frac{R_{c} R}{R_{c}+R} & -\frac{R}{R_{c}+R} & -\left(w_{p 2}+w_{p 3}\right) & -w_{p 2} w_{p 3} & 0 & 0 & 0 & 1 & 0 \\ 0 & 0 & 0 & 0 & 1 & 0 & 0 & 0 & 0 & 0 & 0 \\ 0 & 0 & 0 & 0 & 0 & 1 & 0 & 0 & 0 & 0 & 0 \\ \hline 0 & 0 & \frac{R_{c} R}{R_{c}+R} & \frac{R}{R_{c}+R} & 0 & 0 & 0 & 0 & 0 & 0 & 0\end{array}\right]$

be $47 e^{-3}+s 1.8^{-6}$. From these measurements, the equivalent series resistance (ESR) and inductance of the power supply are estimated at $47 \mathrm{~m} \Omega$ and $1.8 \mathrm{mH}$ respectively.
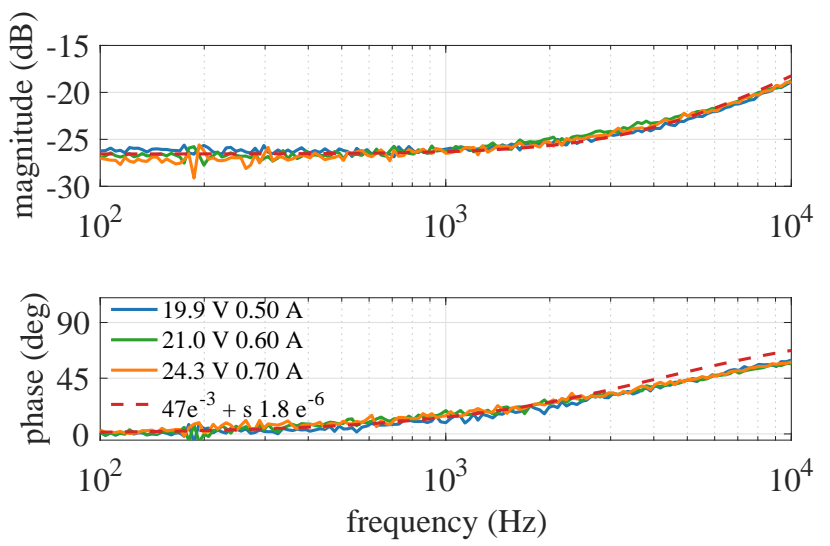

Fig. 6: Output impedance of power supply estimated through curve fitting of experimental measurements

The input filter inductance and capacitance have been measured by means of an impedance analyser. The measurements are depicted in Fig. 7 and 8 respectively. The filter inductance is estimated at $510 \mathrm{mH}$ and after deducting the resistance of the cables used for the measurements of $70 \mathrm{~m} \Omega$, the ESR is estimated as $60 \mathrm{~m} \Omega$, as shown in Fig. 7. The input filter capacitance and ESR are estimated at $95 \mu \mathrm{F}$ and $95 \mathrm{~m} \Omega$ respectively, as depicted in Fig 8. It is to be noted that although second order polynomials provide better approximations, especially at high frequencies, the first order approximation has been selected as it provides sufficient accuracy without unnecessarily complicating the model.

\section{Refined system model}

The last step consists first of experimentally measuring the converter transfer functions, such as the loop gain $T$, the input impedance $Z_{i}$ and the output impedance $Z_{o}$, by means of a network analyser [50]. The experimental measurements are then compared with the corresponding transfer functions obtained from the simulation model. The individual component model and the system model are refined and adjusted iteratively until
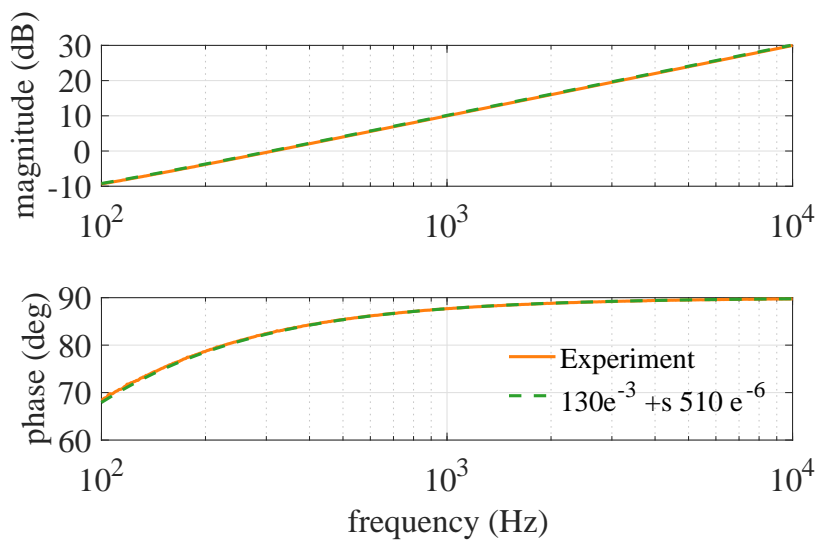

Fig. 7: Input filter inductance including measuring cable resistance estimated through curve fitting of experimental measurements
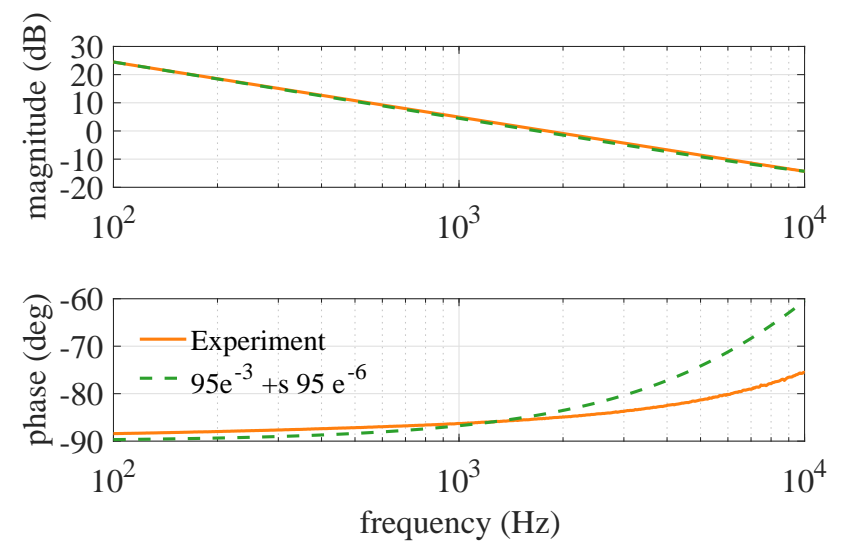

Fig. 8: Input filter capacitance estimated through curve fitting of experimental measurements

a good match is obtained. Fig. 9 and 10 depict the loop gain and the input impedance of the converter obtained from both experiments and the updated simulation model. These measurements have been made without the input filter inductor and with a load of $1.6 \Omega$. The simulation model transfer functions match the experimentally observed behaviour of the 
converter to good accuracy.

Through the process of model refinement, the "initial" values of the system components have been fine-tuned to their final values, as given by the "refined" values in Table I. The "refined" parameter values in Table I are used as nominal values for the equivalent system model (20) in $\mu$ analysis and to run simulations in Simulink ${ }^{\circledR}$ for the case studies in sections V, VI and VII. The "initial" parameter values in Table I are used as nominal values for the case studies in section VIII.

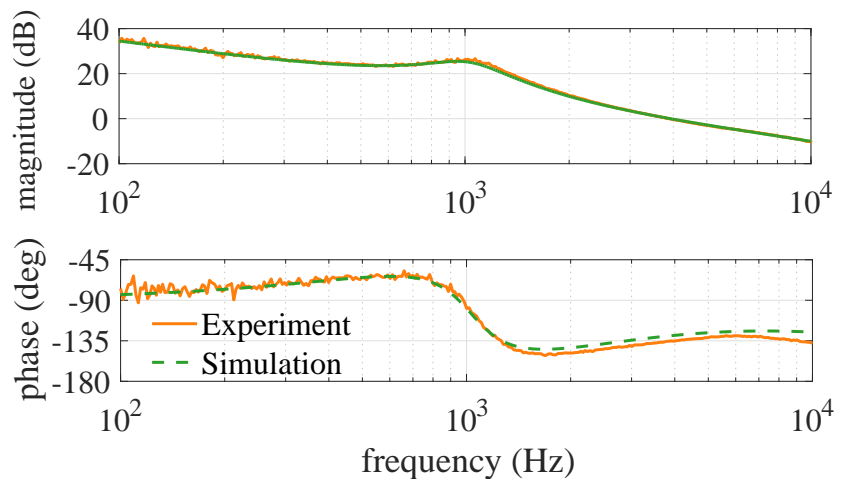

Fig. 9: Validation of simulation model loop gain against experimental measurements of loop gain
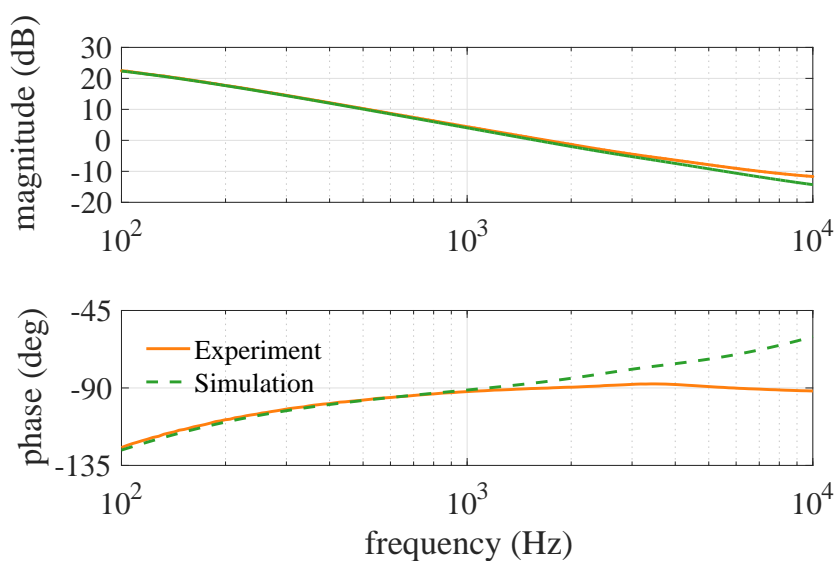

Fig. 10: Validation of simulation model input impedance against experimental measurements of input impedance

\section{LOAD UNCERTAINTY}

In this section, $\mu$ analysis is applied to determine the critical resistive load $R$ that destabilises the system as shown in Fig. 3. In this analysis, referred to as case study 1.1 , the only uncertain parameter is load $R$ that can vary around its nominal value by $\pm 50 \%$ (i.e $R_{v a r}= \pm 50 \%$ as defined in (21) to (23) and Table II). The other system parameters are assumed to be constant with nominal values given as the "refined" values in Table I.

\section{A. $\mu$ analysis}

Prior to $\mu$ analysis, the state space model (20) is converted to its LFT form. In this work, Matlab ${ }^{\circledR}$ Robust Stability
TABLE I: Initial and refined values for the system parameters

\begin{tabular}{|c|c|c|c|}
\hline $\begin{array}{l}\text { Symbol } \\
\text { (units) }\end{array}$ & $\begin{array}{l}\text { Initial } \\
\text { values }\end{array}$ & $\begin{array}{l}\text { Refined } \\
\text { values }\end{array}$ & Description \\
\hline$v_{g}(V)$ & 19.8 & 19.8 & DC source voltage \\
\hline$v_{\text {ref }}(V)$ & 5.1 & 5.1 & Reference Voltage \\
\hline$v_{d}(V)$ & 0.22 & 0.22 & Diode Voltage \\
\hline$v_{o f f}(V)$ & 2.352 & 2.3252 & Offset Voltage \\
\hline$f_{s}(k H z)$ & 51.0 & 51.2 & Switching frequency \\
\hline$f_{m}(-)$ & 0.284 & 0.284 & Modulator gain \\
\hline$R(\Omega)$ & 2.50 & 2.50 & Load resistance \\
\hline$R_{\text {on }}(m \Omega)$ & 160 & 160 & Switch-on resistance \\
\hline$R_{d}(m \Omega)$ & 12 & 12 & Diode on-resistance \\
\hline$R_{\text {in }}(m \Omega)$ & 135 & 160 & Input Resistance \\
\hline$L_{\text {in }} \mu H()$ & 480 & 511.8 & Input filter inductance \\
\hline$C_{i n}(\mu F)$ & 100 & 95 & Input filter capacitance \\
\hline$R_{\text {cin }}(m \Omega)$ & 80 & 95 & ESR of input filter capacitor \\
\hline$L(\mu H)$ & 42 & 45.5 & Output filter inductance \\
\hline$R_{l}(m \Omega)$ & 45 & 50 & ESR of output filter inductor \\
\hline$C(\mu F)$ & 590 & 540 & Output filter capacitance \\
\hline$R_{c}(m \Omega)$ & 10 & 17 & ESR of output filter capacitor \\
\hline$R_{1}(k \Omega)$ & 20.0 & 19.9 & Resistance in compensator \\
\hline$R_{2}(k \Omega)$ & 20.0 & 19.7 & Resistance in compensator \\
\hline$R_{3}(k \Omega)$ & 2.0 & 2.0 & Resistance in compensator \\
\hline$C_{1}(n F)$ & 8.22 & 8.22 & Capacitance in compensator \\
\hline$C_{2}(n F)$ & 4.72 & 4.72 & Capacitance in compensator \\
\hline$C_{3}(n F)$ & 0.331 & 0.331 & Capacitance in compensator \\
\hline
\end{tabular}

TABLE II: Case 1.1 - Uncertain resistive load

\begin{tabular}{ccc}
\hline Parameter & $\begin{array}{c}\text { Nominal } \\
\text { value }\left(R_{o}\right)\end{array}$ & $\begin{array}{c}\text { Range of variation }\left(R_{\text {var }}\right) \\
\text { with respect to nominal value }\end{array}$ \\
\hline $\mathrm{R}$ & $2.5 \Omega$ & $\pm 50 \%$
\end{tabular}

Toolbox has been employed for performing both LFT and SSV analysis. Expressing (20) in the LFT form requires that all uncertain parameters be first converted to their LFT forms. In this case study, the uncertain element $R$ has to be expressed as a function of its normalised form $\delta_{R}$ which lies between -1 and 1 as shown in (21). $R_{o}$ and $R_{v a r}$ can be derived from the minimum value $\left(R_{\min }\right)$ and the maximum value $\left(R_{\max }\right)$ of the resistive load as shown in (22) and (23) [48].

$$
\begin{aligned}
& R=R_{o}+R_{o} R_{\text {var }} \delta_{R} \quad \text { where } \delta_{R} \in[-1,1] \\
& R_{o}=\left(R_{\text {max }}+R_{\text {min }}\right) / 2 \\
& R_{\text {var }}=\left(R_{\text {max }}-R_{\text {min }}\right) /\left(R_{\max }+R_{\min }\right)
\end{aligned}
$$

The normalised parameters $\delta_{R}$ are then extracted from the uncertain system model (20) and grouped in a diagonal matrix in a feedback form by applying LFT technique. The resulting uncertainty matrix is shown in (24). $\delta_{R}$ appears 227 times in the $\Delta$ matrix, which corresponds to the number of times $R$ appears in the system matrix.

$$
\Delta(j \cdot 2 \pi \cdot f)=\delta_{R} I_{227 \times 227}
$$

$\mu$ analysis is then applied to the uncertain system model in LFT form by using Matlab ${ }^{\circledR}$ Robust Stability Toolbox. The results are shown in Fig. 11a and 11b.

For this test, $\mu$ is equal to 1.44 as given by the peak value of the charts in Fig. 11a and 11b. The smallest destabilising 


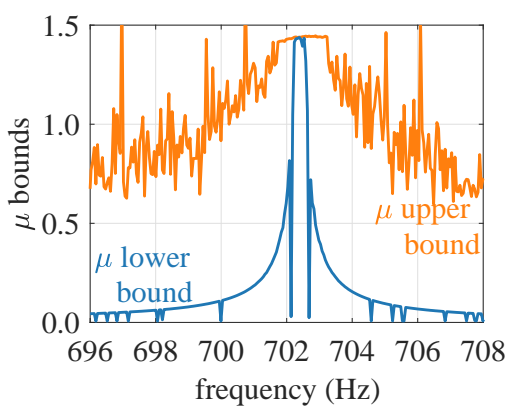

(a)

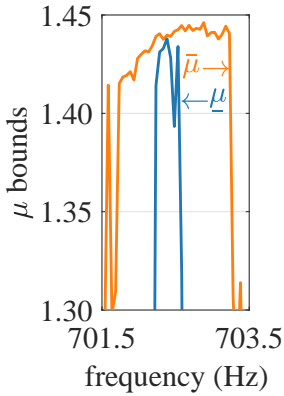

(b)
Fig. 11: Case 1.1 - System with load uncertainty (a) $\mu$ chart to predict critical $R$ (b) zoomed area near the peak of the $\mu$ chart

matrix, of size $227 \times 227$ as given by (25), gives the robust stability margin $(1 / \mu)$ as 0.696 [49].

$$
\Delta(j \cdot 2 \pi \cdot 702.4)=-0.696 I_{227 \times 227}
$$

By comparing (25) with (24), it can be noted that $\delta_{R}=$ -0.696 . The critical destabilising load can be computed from $\delta_{R}=-0.696$ and (21) and is equal to $1.63 \Omega$ or $16.0 \mathrm{~W}$, as shown in (26) and Table III.

$$
\begin{aligned}
R & =R_{o}+R_{o} R_{\text {var }} \delta_{R} \quad \text { where } \delta_{R} \in[-1,1] \\
& =2.5+2.5 \times 50 \% \times(-0.696) \\
& =2.5-2.5 \times 35 \%=1.63
\end{aligned}
$$

TABLE III: Case 1.1 - System with load uncertainty, $\mu$ analysis results

\begin{tabular}{ccccc}
\hline $\begin{array}{c}\text { Perturbation } \\
\text { matrix }\end{array}$ & $\begin{array}{c}\text { Robust stability } \\
\text { margin }(1 / \mu)\end{array}$ & $\mu$ & \multicolumn{2}{c}{ Critical load } \\
& & & power $(W)$ \\
\hline$\Delta(j \cdot 2 \pi \cdot 702.4)$ & 0.696 & 1.44 & $1.63 \Omega$ & $16.0 \mathrm{~W}$
\end{tabular}

$\mu>1$ indicates that the system is not robustly stable, i.e. the system does not remain stable over the whole uncertainty range between $1.25 \Omega$ and $3.75 \Omega$, as defined in Table II. In order to ensure that the system remains robustly stable, $\mu$ should be less than 1 . This can be achieved by scaling the operating range of the system load by $1 / \mu$, the robust stability margin. The robust uncertainty range of $R$ is thus $2.5 \pm 2.5 \times$ $50 \% \times 0.696$ i.e. $2.5 \Omega \pm 35 \%$.

\section{B. Experimental results}

In this experiment, the electronic resistive load $R$ was decreased in small steps from a peak value of $2 \Omega$ until the system reached boundary stability. When $R$ was decreased to $1.62 \Omega$ at $t=0.453 \mathrm{~s}$ (i.e. $I_{o}$ increased to $3.15 \mathrm{~A}$ ), the system reached the boundary condition of stability, as shown by the sustained oscillations in $V_{i n}$ and $V_{o}$ in Fig. 12a and 12b. When $R$ was increased back to $2 \Omega$ at $t=0.703 s$ (i.e. $I_{o}$ decreased to $2.55 \mathrm{~A}$ ), the system stabilised again, as shown in Fig. 12a. The critical load resistance of $1.62 \Omega$ closely matches the value of $1.63 \Omega$ predicted by $\mu$ analysis, as depicted in Table IV, with an error of $0.6 \%$. It should be noted that, although the experimental results match closely the $\mu$ prediction, they are subject to measurement error.

In addition, simulation was ran in the Simulink ${ }^{\circledR}$ environment on the refined nonlinear model of the buck converter system under study. The simulation result of the critical load, as given in Table IV, fall within $1.2 \%$ of the $\mu$ prediction, and the discrepancy may be accounted for by uncertainties in the model such as the approximation error of duty cycle $D$. Model uncertainties can be considered in the robust stability analysis, as is demonstrated later in section VIII.

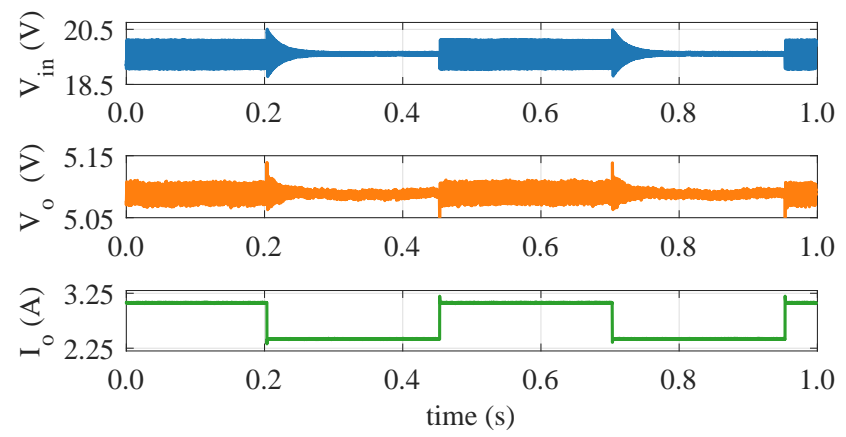

(a)
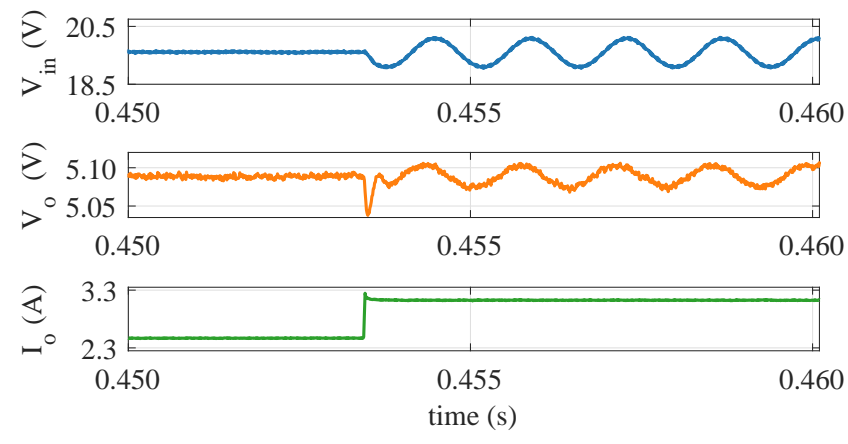

(b)

Fig. 12: Case 1.1 - Experimental results for the system with load uncertainty (a) system is at boundary of stability with $R=1.63 \Omega$ from $t=0.453 s$ to $0.703 s$ (b) zoomed area near $t=0.453 \mathrm{~s}$

TABLE IV: Case 1.1 - System with load uncertainty, $\mu$ analysis and experimental results

\begin{tabular}{cccc}
\hline $\begin{array}{c}\text { Uncertain } \\
\text { load }\end{array}$ & $\mu$ analysis & Critical values \\
& Simulation & Experiment \\
\hline$R(\Omega)$ & $1.63 \Omega$ & $1.61 \Omega$ & $1.62 \Omega$ \\
power $(W)$ & $16.0 W$ & $16.1 W$ & $16.1 W$
\end{tabular}

\section{Computation time}

In order to assess the effect of the duty cycle approximation on robust stability margin and computation time, $\mu$ analysis has also been performed by using the zeroth order approximation of the duty cycle $D$, referred to as case study 1.2 in Table V. The analyses, with zeroth and first order 
approximation of the duty cycle $D$, have been performed using a frequency grid of 250 points between $650 \mathrm{~Hz}$ and $750 \mathrm{~Hz}$ on an Intel Core i7-3820 $3.6 \mathrm{GHz}$ processor with 32GB RAM. The $\mu$ results and the computation times are given in Table V. The zeroth order approximation introduces an error of $7 \%$ in the robust stability margin and an error of $4 \%$ in the critical load $R$, with respect to the first order approximation. Inspite of the loss in accuracy, the computation time is seen to reduce considerably, as noted in Table $\mathrm{V}$. This is due to the fact that $D$ appears 189 times in the system model. By setting $D$ as a constant, the size of the uncertainty matrix reduces from $227 \times 227$ to $37 \times 37$. It can be inferred that the size of the uncertainty matrix has a key influence on computational cost. Of note is that nonlinear terms can be treated as uncertain elements of the system, as will be examined in section VIII.

TABLE V: Cases 1.1 and 1.2 - System with load uncertainty, evaluation of polynomial approximations of duty cycle $D$

\begin{tabular}{cccccc}
\hline $\begin{array}{c}\text { Case } \\
\text { Study }\end{array}$ & $\begin{array}{c}D \\
\text { approximation }\end{array}$ & $\begin{array}{c}\text { Robust } \\
\text { stability } \\
\text { margin }(1 / \mu)\end{array}$ & $\begin{array}{c}\text { Critical } \\
\text { load }\end{array}$ & $\begin{array}{c}\text { Size } \\
\Delta(j w)\end{array}$ & $\begin{array}{c}\text { Computation } \\
\text { time }\end{array}$ \\
\hline & & & & & \\
1.1 & $1^{s t}$ order & 0.696 & $1.63 \Omega$ & $227 \times 227$ & $34 \mathrm{~min}$ \\
1.2 & $0^{t h}$ order & 0.745 & $1.57 \Omega$ & $37 \times 37$ & $54 \mathrm{~s}$
\end{tabular}

\section{LOAD AND LINE RESISTANCE UNCERTAINTIES}

In practice, the actual parameter values of system components may differ at the assembly stage when compared to initial design values. These parametric uncertainties may lead to system instability. This section considers the case when the load and the line resistance of the analysed system are not known accurately, but are known to lie within certain ranges, as defined in Table VI. The $\mu$ tool can be used to determine the bounds within which these uncertain parameters must lie in order to guarantee stability of the system under study. The other system parameters are fixed and given by the "refined" values in Table I.

TABLE VI: Case 2.1 - Uncertain load and line resistance

\begin{tabular}{ccc}
\hline $\begin{array}{c}\text { Uncertain } \\
\text { parameters }\end{array}$ & $\begin{array}{c}\text { Nominal } \\
\text { value }\end{array}$ & $\begin{array}{c}\text { Range of variation } \\
\text { with respect to nominal value }\end{array}$ \\
\hline$R$ & $R_{o}=2.5 \Omega$ & $R_{\text {var }}= \pm 50 \%$ \\
$R_{\text {in }}$ & $R_{\text {ino }}=0.3 \Omega$ & $R_{\text {invar }}= \pm 50 \%$
\end{tabular}

\section{A. $\mu$ analysis}

$\mu$ analysis is performed on the system model (20), by using the first order Taylor series approximation of the duty cycle $D$ with respect to both $R$ and $R_{i n}$. The peak value of $\mu$ is equal to 1.24 , and occurs at a frequency of $701.3 \mathrm{~Hz}$, as can be seen in Fig. 13a and 13b. The robust stability margin $(1 / \mu)$ is 0.803 . The general uncertainty matrix is given by (27) and the critical uncertainty matrix obtained from $\mu$ analysis is shown in (28). By comparing (27) and (28), $\delta_{R}$ and $\delta_{R i n}$ are found to be equal to -0.803 . The critical values of $R$ and $R_{i n}$ can be computed as $1.50 \Omega$ and $1.18 \Omega$ respectively, from the aforementioned $\delta_{R}$ and $\delta_{R i n}$ values and the general equation
(1). This case study shows that if the values of $R$ and $R_{\text {in }}$ are kept within $80.3 \%$ of their respective nominal values, the system is ensured to be robustly stable. It can be noted that the robust stability margin in case 2.1 is increased with respect to case 1.1 , as the line resistance $R_{\text {in }}$ is set in the range [150 $m \Omega, 450 \mathrm{~m} \Omega]$, and in that can provide more damping to the input $L C$ filter.

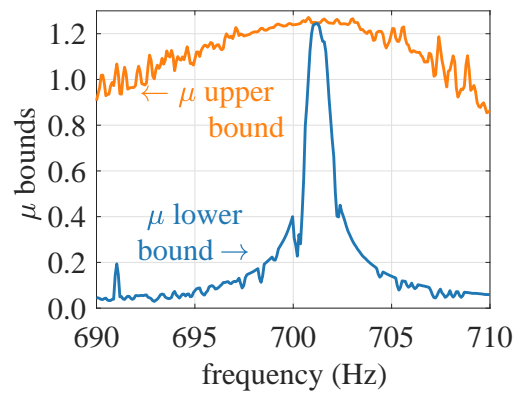

(a)

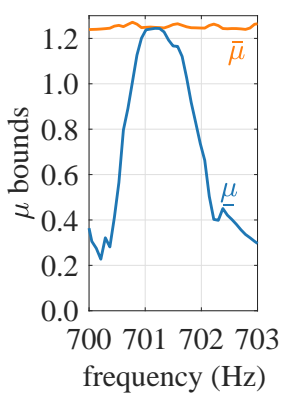

(b)
Fig. 13: Case 2.1 - System with load and line resistance uncertainties (a) $\mu$ chart to predict critical load $R$ (b) zoomed area near the peak of the $\mu$ chart

$$
\begin{array}{r}
\Delta(j \cdot 2 \pi \cdot f)=\operatorname{diag}\left(\delta_{R} I_{227}, \delta_{R i n} I_{217}\right) \\
\Delta(j \cdot 2 \pi \cdot 701.3)=\operatorname{diag}\left(-0.803 I_{227}, \quad-0.803 I_{217}\right)
\end{array}
$$

\section{B. Experimental results}

In order to validate the $\mu$ predictions of the case study 2.1 , a set of experiments were performed on the buck converter system. By keeping $R_{i n}$ around $0.18 \Omega$, the load was slowly decreased until the system became unstable. The experimental results are shown in Fig. 14. When $R$ is set at $1.89 \Omega$, the system stabilises, as shown in the top chart in Fig. 14. When $R$ is decreased to $1.54 \Omega$, the system reaches boundary stability, as shown by the sustained oscillations in the input voltage $V_{i n}$ in the middle chart in Fig. 14. Decreasing $R$ to $1.50 \Omega$ causes the system to become unstable, as shown in the bottom chart in Fig. 14. The line resistance $R_{i n}$, used in the experiment, has been accurately measured and found to be $1.85 \Omega$. The experimental results, summarised in Table VII, are in close agreement with the $\mu$ predictions, with an error falling within $3 \%$, and the discrepancy may be accounted for by measurement error and some uncertainties in the system model.

It is to be added that the Simulink ${ }^{\circledR}$ model of the system, which is based on the state space model used in $\mu$ analysis, has also been used to verify the $\mu$ analysis results. The simulation results from the Simulink ${ }^{\circledR}$ model closely match the $\mu$ predictions as shown in Table VII, and the discrepancy may be accounted for by some uncertainties in the model such as the approximation error of duty cycle D. The effect of model uncertainties on the system stability margins is analysed in section VIII.

\section{TEMPERATURE UNCERTAINTY}

Although, a power system can be modelled to good accuracy, however, in practice, the values of its system components 


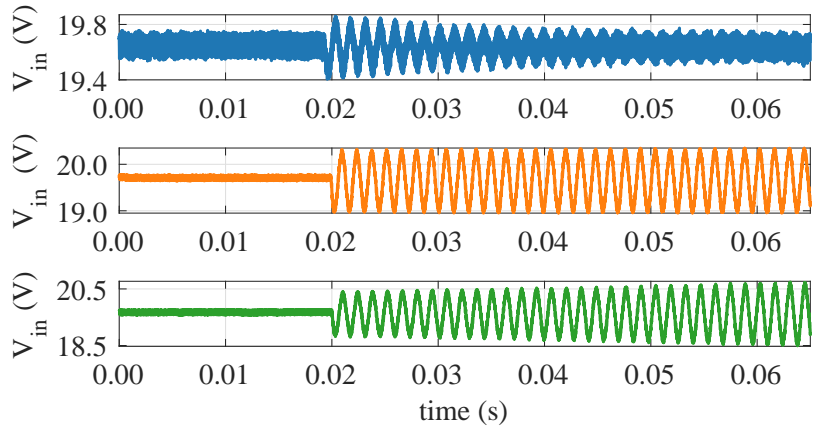

Fig. 14: Case 2.1 - Experimental results for system with uncertain $R$ with $R_{\text {in }}=185 \mathrm{~m} \Omega$ (i) top chart: system is stable with $R=1.89 \Omega$ (ii) middle chart: system is at boundary of stability with $R=1.54 \Omega$ (iii) bottom chart: system is unstable with $R=1.50 \Omega$

TABLE VII: Case 2.1 - System with load and line resistance uncertainties, $\mu$ analysis, simulation and experimental results

\begin{tabular}{cccc}
\hline Uncertain & \multicolumn{3}{c}{ Critical values } \\
parameters & $\mu$ analysis & Simulation & Experiment \\
\hline & & & \\
$R(\Omega)$ & 1.50 & 1.49 & 1.54 \\
$R_{\text {in }}(\Omega)$ & 0.180 & 0.179 & 0.185
\end{tabular}

are bound to vary during operation. Temperature is one of the main factors that can introduce uncertainties in multiple system parameters. In this section, the $\mu$ approach is applied to the buck converter, as shown in Fig. 3, when it is exposed to large temperature variation, based on the methodology used in section $\mathrm{V}$. The duty cycle $D$ is estimated by its zeroth order approximation. The $\mu$ predictions are thereafter evaluated against the $\mu$ results from case study 1.2 in section $\mathrm{V}$ in order to investigate the effect of extreme temperature variation on the robust stability margin.

\section{A. Uncertain parameters}

In this analysis, referred to as case study 3.1, the buck converter is considered to be working in an environment where the temperature may vary between $-40{ }^{\circ} \mathrm{C}$ and $80^{\circ} \mathrm{C}$ with a reference value of $20^{\circ} \mathrm{C}$, as shown in Table VIII.

The variations in temperature may influence the characteristics of the resistive components of the buck converter, which include but are not limited to the system parasitics and cable resistances. These components, which are denoted as $R_{e s}$ further in the text and comprising $R_{i n}, R_{c i n}, R_{l}, R_{o n}$, $R_{c}$, may have different temperature coefficients depending on their constituent materials. However, for the sake of simplicity, a temperature coefficient of resistance $(\alpha)$ of $0.004 /{ }^{\circ} \mathrm{C}$ as for a copper wire is assumed for the aforementioned components in this study. From (29) and Table VIII, it can be seen that the variations in temperature (T) of $\pm 60{ }^{\circ} \mathrm{C}$ cause variations in the resistive components of $\pm 24 \%$ around their nominal values denoted as $\operatorname{Res}_{0}$.

$$
R_{\text {esvar }}=\left(R_{\text {es }}-R_{\text {eso }}\right) / R_{\text {eso }}=\alpha \Delta T
$$

The nominal values of the system components, including $R_{e s}$, are given as the "refined" values in Table I. It is assumed that the resistive load varies within $\pm 50 \%$ of its nominal value, as in case 1.1 in section V, and as depicted in Table VIII. The $\mu$ approach is applied to study the effect of the defined temperature variation on stability robustness of the analysed system.

TABLE VIII: Case 3.1 - Uncertainties in load and temperature

\begin{tabular}{lcc}
\hline $\begin{array}{l}\text { Uncertain } \\
\text { parameters }\end{array}$ & $\begin{array}{c}\text { Nominal } \\
\text { value }\end{array}$ & $\begin{array}{c}\text { Range of variation } \\
\text { with respect to nominal val }\end{array}$ \\
$T$ & $T_{o}=20^{\circ} \mathrm{C}$ & $\Delta T= \pm 60^{\circ} \mathrm{C}$ \\
$R_{\text {es }}$ & $R_{\text {eso }}$ & $R_{\text {esvar }}=\alpha \Delta \mathrm{T}= \pm 24 \%$ \\
$R$ & $R_{o}=2.5 \Omega$ & $R_{\text {var }}= \pm 50 \%$
\end{tabular}

\section{B. $\mu$ analysis}

By employing LFT technique, the system model (20) is first expressed in the LFT form. The structure of the resulting uncertainty matrix is shown in (30).

$$
\begin{aligned}
& \Delta(j \cdot 2 \pi \cdot f)=\operatorname{diag}\left(\delta_{R} I_{41}, \delta_{R c} I_{13}, \delta_{R c i n} I_{38},\right. \\
& \left.\delta_{R i n} I_{25}, \delta_{R l} I_{23}, \delta_{R o n} I_{27}\right)
\end{aligned}
$$

The $\mu$ approach is then applied to the uncertain system model in its LFT form. The $\mu$ chart is depicted in Fig. 15 where the peak of the $\mu$ lower bound is seen to be equal to 1.98 at the critical frequency of $704 \mathrm{~Hz}$.

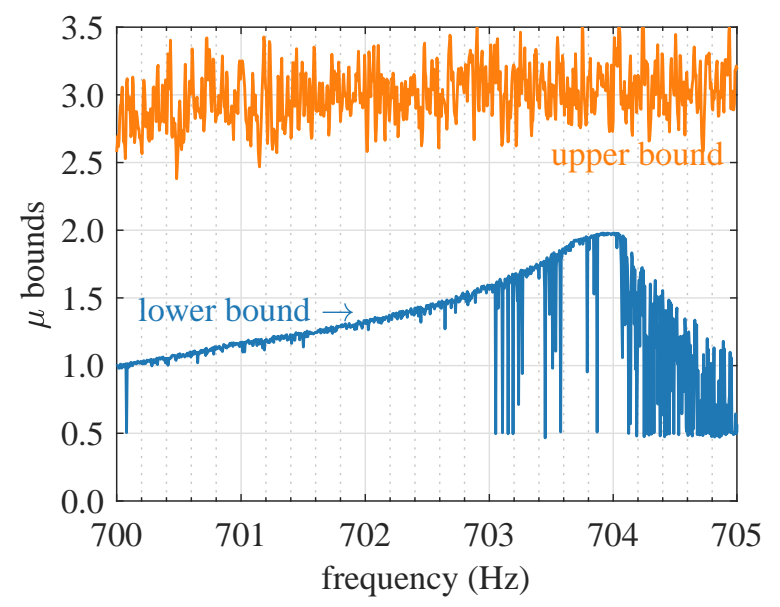

Fig. 15: Case 3.1 - System with load and temperature uncertainties, $\mu$ chart to predict load $R$ and critical resistive components $R_{e s}$

The corresponding critical values of the resistive load and resistive components, calculated from the $\mu$ bounds, are given in Table $\mathrm{X}$. The critical destabilising load is now $1.87 \Omega$ $(13.9 \mathrm{~W})$, based on the $\mu$ lower bound. This represents a robust stability margin of 0.505 , as shown in Table IX.

\section{Simulation verification}

The refined Simulink ${ }^{\circledR}$ model of the buck converter is used to verify the $\mu$ analysis results obtained in the earlier 
TABLE IX: System with load and temperature uncertainty $\mu$ analysis results for load

\begin{tabular}{|c|c|c|c|c|}
\hline \multirow{2}{*}{$\begin{array}{l}\text { Uncertain } \\
\text { parameter }\end{array}$} & \multirow{2}{*}{$\begin{array}{l}\text { Robust stability } \\
\text { margin }\end{array}$} & \multirow[t]{2}{*}{$\mu$} & \multicolumn{2}{|c|}{ Critical load } \\
\hline & & & $R(\Omega)$ & power $(W)$ \\
\hline Load & 0.505 & 1.98 & $1.87 \Omega$ & $13.9 \mathrm{~W}$ \\
\hline
\end{tabular}

subsection. When the critical values predicted by $\mu$ analysis, as given in Table $X$, are input in the Simulink ${ }^{\circledR}$ model of the buck converter, the system reaches boundary stability. Fig. 16a and 16b show the results for the case where the load $R$ is varied with the other $R_{e s}$ components fixed at their critical values given in Table $\mathrm{X}$.

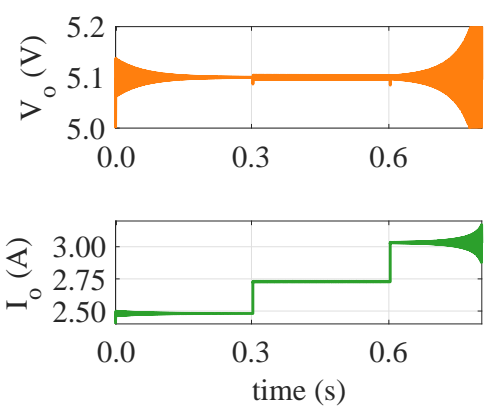

(a)
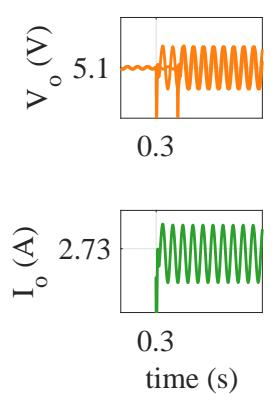

(b)
Fig. 16: Case 3.1- System with load and temperature uncertainties (a) top figure - voltage $v_{o}$, bottom figure - current $i_{o}$ (i) at $t=0 \mathrm{~s}, R=1.1 \times 1.87 \Omega$ (ii) at $t=0.3 \mathrm{~s}, R=1.87$ $\Omega$ (iii) at $t=0.6 \mathrm{~s}, R=0.9 \times 1.87 \Omega$, where $R=1.87 \Omega$ is the critical load predicted by $\mu$ analysis (b) zoomed area near $t=0.3 \mathrm{~s}$

When $R$ is set to be $10 \%$ higher than its critical value of $1.87 \Omega$ at $t=0 s$ (i.e. $I_{o}$ is $2.48 A$ ), the system stabilises as shown in Fig. 16a. When $R$ is decreased to the critical value of $1.87 \Omega$ at $t=0.3 s$ (i.e. $I_{o}$ is $2.73 A$ ), the system reaches boundary stability condition, as can be seen from the sustained oscillations in $V_{o}$ in Fig. 16a and 16b. When $R$ is decreased by a further $10 \%$ below $1.87 \Omega$ at $t=0.6 s\left(I_{o}\right.$ is $\left.3.03 A\right)$, the system becomes unstable. The simulation results closely match the results predicted by $\mu$ analysis as shown in Table $\mathrm{X}$.

\section{Results analysis}

From robust stability analysis, $\mu>1$ indicates that the system under investigation, with $50 \%$ load uncertainty, is not guaranteed to remain stable over the whole range of variation of temperature (i.e. $-40{ }^{\circ} \mathrm{C}$ to $80^{\circ} \mathrm{C}$ ). Based on the examination of the hypercube concept presented in section II, the buck converter system under study remains robustly stable within a hypercube of the sixth-order dimension, of coordinate size $\mu=0.505$, and centred about the nominal point. Any combination of parameter values chosen within the aforementioned hypercube will ensure the robust stability of the buck converter under study, within the range of temperature variation defined in Table VIII. As depicted in Table XI, when the uncertainty in temperature is considered, the robust stability margin is 0.505 . When temperature variation is neglected, the robust stability margin is 0.745 , as shown in case study 1.2 and Table V. This represents a $32 \%$ decrease in the robust stability margin, as shown in Table XI. The duty cycle $D$ is constant in both cases 3.1 and 2.1. This study confirms that the variation in temperature can have a significant influence on robust stability margin and must therefore be incorporated in the stability assessment.

TABLE X: Case 3.1 - System with load and temperature uncertainties, $\mu$ analysis and time domain simulation results

\begin{tabular}{lccc}
\hline $\begin{array}{l}\text { Uncertain } \\
\text { parameters }\end{array}$ & $\begin{array}{c}\text { Nominal } \\
\text { value }\end{array}$ & \multicolumn{2}{c}{ Critical values } \\
$\mu$ analysis & Simulation \\
\hline$R(\Omega)$ & 2.50 & 1.87 & 1.87 \\
$R$ & 17 & 14.9 & 14.9 \\
$R_{c}(m \Omega)$ & 95 & 83.5 & 83.5 \\
$R_{\text {cin }}(m \Omega)$ & 160 & 140.6 & 140.6 \\
$R_{\text {in }}(m \Omega)$ & 160 & 43.9 & 43.9 \\
$R_{l}(m \Omega)$ & 50 & 154.7 & 154.7 \\
$R_{\text {on }}(m \Omega)$ & 160 & &
\end{tabular}

TABLE XI: Cases 3.1 and 1.2 - Effect of temperature on robust stability margin with $D$ constant

\begin{tabular}{|c|c|c|c|c|}
\hline Case & Temperature & \multicolumn{2}{|c|}{ Critical load } & Robust stability \\
\hline Study & Considered & $R(\Omega)$ & power $(W)$ & Margin \\
\hline 3.1 & Yes & $1.87 \Omega$ & $13.9 W$ & 0.505 \\
\hline 1.2 & No & $1.57 \Omega$ & $16.6 \mathrm{~W}$ & 0.745 \\
\hline
\end{tabular}

\section{Model UnCERTAinties}

In practice, it is neither viable nor time-efficient to create highly refined system models to represent actual systems. Hence, approximate system models, with a good trade-off between accuracy and simplicity, are often used for design. The nominal values of their system components are generally based on known data such as nameplate information. This section aims to demonstrate how model uncertainties, which may be known to different level of accuracy, can be incorporated in robust stability analysis without compromising the reliability of the results. In addition, it examines the effect of model uncertainties on robust stability margin.

The approach is illustrated by applying it to the buck converter example power system in Fig 3. The "initial" values of the system components, as given in Table I, are used as the nominal values for $\mu$ analysis. Three cases are investigated, as defined in Table XII. In case 4.1, robust stability margin is evaluated without taking into account any uncertainties in the model. Only uncertainty in the load is considered. In cases 4.2 and 4.3, uncertainties in the model are included in the analyses. However, the approximation errors in the model in case 4.2 are larger as compared with those in case 4.3. The other system parameters are considered to be fixed as defined by the "initial" parameter values in Table I. 
TABLE XII: Cases 4.1, 4.2, 4.3 - Uncertainties in load and system model

\begin{tabular}{lrrrr}
\hline Uncertain & Nominal & \multicolumn{3}{c}{ Range of variation } \\
parameters & value & Case 4.1 & Case 4.2 & Case 4.3 \\
\hline & & & & \\
$R$ & $2.50 \Omega$ & $\pm 50 \%$ & $\pm 50 \%$ & $\pm 50 \%$ \\
$R_{i n}$ & $135 m \Omega$ & - & $\pm 50 \%$ & $\pm 30 \%$ \\
$L_{i n}$ & $480 \mu H$ & - & $\pm 50 \%$ & $\pm 30 \%$ \\
$C_{i n}$ & $100 \mu F$ & - & $\pm 10 \%$ & $\pm 6 \%$ \\
$R_{c i n}$ & $80 m \Omega$ & - & $\pm 10 \%$ & $\pm 6 \%$ \\
$L$ & $42 \mu H$ & - & $\pm 50 \%$ & $\pm 30 \%$ \\
$R_{l}$ & $45 m \Omega$ & - & $\pm 10 \%$ & $\pm 6 \%$ \\
$C$ & $590 \mu F$ & - & $\pm 50 \%$ & $\pm 30 \%$ \\
$R_{c}$ & $10 m \Omega$ & - & $\pm 10 \%$ & $\pm 6 \%$ \\
$D$ & 0.2768 & - & $\pm 4.5 \%$ & $\pm 4.5 \%$
\end{tabular}

\section{A. System with no model uncertainty}

This subsection evaluates the robust stability margin for case 4.1. $\mu$ analysis is applied to the system model (20) with the uncertain parameters as defined in Table XII. The $\mu$ chart, as depicted in Fig. 17a and 17b, shows that the $\mu$ lower bound is 1.63. This corresponds to a robust stability margin of 0.614 and a critical load of $1.73 \Omega$ or $15 \mathrm{~W}$, as shown in Table XIII. The buck converter is predicted to remain stable for an output power of up to $15 \mathrm{~W}$.

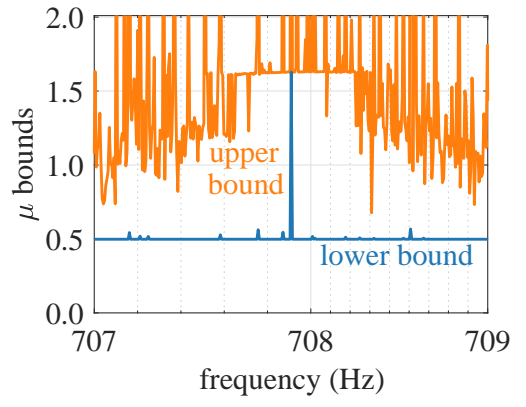

(a)

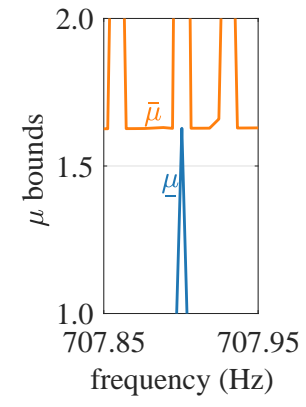

(b)
Fig. 17: Case 4.1: System with load uncertainty and no model uncertainty (a) $\mu$ chart to predict critical load $R$ (b) zoomed area near the peak of the $\mu$ chart

TABLE XIII: Case 4.1 - System with load uncertainty and no model uncertainty, $\mu$ analysis results for load

\begin{tabular}{ccccc}
\hline $\begin{array}{c}\text { Uncertain } \\
\text { parameter }\end{array}$ & $\begin{array}{c}\text { Robust stability } \\
\text { margin }\end{array}$ & $\underline{\mu}$ & \multicolumn{2}{c}{ Critical load } \\
power $(W)$ \\
\hline Load & 0.614 & 1.63 & $1.73 \Omega$ & $15.0 \mathrm{~W}$
\end{tabular}

\section{B. System with model uncertainties}

The stability robustness for cases 4.2 and 4.3 are evaluated in this subsection. The maximum possible errors that may be expected in the nominal values, as given in Table XII, are taken into account in the analyses. This is based on the knowledge of the system. For instance, for case 4.2, the tolerances of the capacitors and inductors are known to be well within $10 \%$ of their nominal values. Parasitic elements, which may be nonlinear in nature, are generally hard to quantify. Hence, the system parasitics such as the ESR of the inductors and capacitors, have been considered to vary within a maximum range of $\pm 50 \%$ within their estimated nominal values. Further, nonlinear terms in the system model may be treated as uncertain elements. Hence in this case, the duty cycle $D$ is set as an uncertain parameter with $4.5 \%$ uncertainty, based on its maximum variation range as depicted in Fig. 5. This eliminates the need for high order approximations, which has the added advantage of reducing the size of the uncertainty matrix. As in previous case studies, the load $R$ is considered to vary within $50 \%$ of its nominal value. In case 4.3 , it is considered that the values of the uncertain parameters are known with better accuracy, with variation ranges of the system inductances, capacitors and ESRs being tighter, as depicted in Table XII.

$\mu$ analysis is applied to the system model (20) based on the uncertain parameters defined in Table XII, for cases 4.2 and 4.3. Following the LFT operation, the structure of the uncertainty matrix, of size $351 \times 351$, is obtained as (31).

$$
\begin{array}{r}
\Delta(j \cdot 2 \pi \cdot f)=\operatorname{diag}\left(\delta_{C} I_{2}, \delta_{C i n} I_{6}, \delta_{D} I_{189}, \delta_{L} I_{4}, \delta_{L i n} I_{8},\right. \\
\\
\left.\delta_{R} I_{41}, \delta_{R c} I_{13}, \delta_{R c i n} I_{39}, \delta_{R i n} I_{25}, \delta_{R l} I_{24}\right)
\end{array}
$$

The $\mu$ charts for cases 4.2 and 4.3 are shown in Fig. 18a and Fig. 18b respectively. The $\mu$ lower bound is 4.76 for case 4.2. This corresponds to a robust stability margin of 0.21 and a critical load of $2.24 \Omega$ or $11.6 \mathrm{~W}$, as depicted in Table XIV. For case 4.3 , the $\mu$ lower bound is 3.47 . The associated robust stability margin is 0.288 , and the critical load is $2.14 \Omega$ or $12.2 \mathrm{~W}$. The results are shown in Table XIV. Thus after taking into account the aforementioned uncertainties in the model, the buck converter is predicted to remain stable for an output power of up to $11.6 \mathrm{~W}$ for case 4.2 , and up to $12.2 \mathrm{~W}$ for case 4.3, as shown in Table XIV. The results for case 4.1 are included in Table XIV for completion.

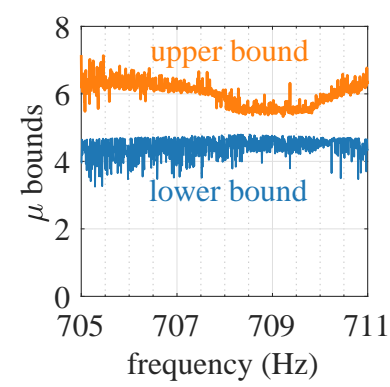

(a)

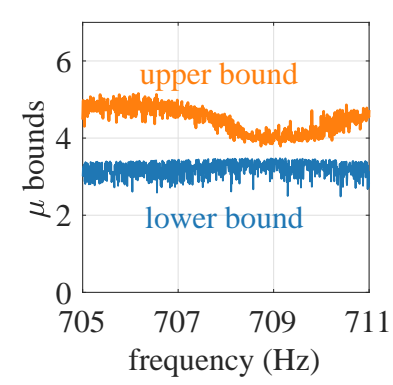

(b)
Fig. 18: Cases 4.2 and 4.3 - System with load and model uncertainties, $\mu$ charts to predict critical load $R$ for (a) case 4.2 (b) case 4.3

With the aim of verifying the $\mu$ results, the eigenvalues of the nominal model of the linear equivalent model (20) are evaluated against the eigenvalues of the critical linear equivalent model for case 4.2, obtained from the critical parameter values predicted by $\mu$ analysis. The corresponding 
TABLE XIV: Cases 4.1, 4.2, 4.3 - System with load and model uncertainties - $\mu$ analysis results for load

\begin{tabular}{cccccc}
\hline $\begin{array}{c}\text { Case } \\
\text { study }\end{array}$ & $\begin{array}{c}\text { Model } \\
\text { uncertainties }\end{array}$ & $\begin{array}{c}\text { Robust stability } \\
\text { margin }\end{array}$ & $\underline{\mu}$ & \multicolumn{2}{c}{ Critical load } \\
power $(W)$
\end{tabular}

plots of the eigenvalues are depicted in Fig. 19a and 19b. It can be noted that applying the critical values, predicted by $\mu$ analysis, brings the eigenvalues near to the imaginary axis. This confirms that $\mu$ analysis has identified the critical values at the boundary of stability.

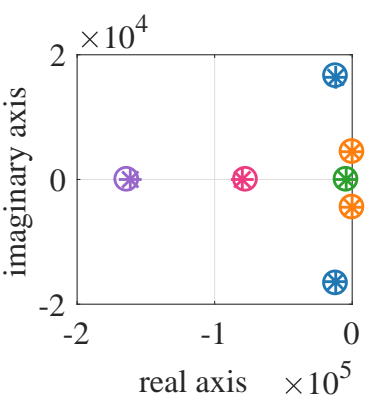

(a)

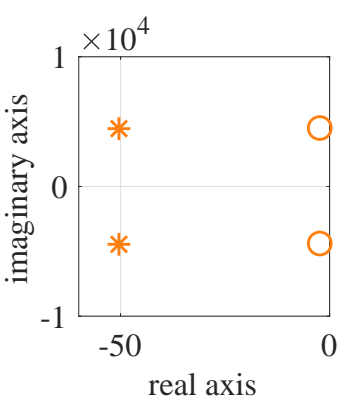

(b)
Fig. 19: Case 4.2: System with load and model uncertainties (a) plot of eigenvalues based on $\mu$ lower bound predictions, (*) eigenvalues with nominal parameters, (o) eigenvalues with critical parameters (b) zoomed view near the imaginary axis

\section{Results analysis}

The robust stability margins $(1 / \mu)$ for cases $4.1,4.2$ and 4.3 are depicted in Table XIV. It is interesting to note that the stability robustness for cases $4.1,4.2$ and 4.3 is represented by hypercubes, of the tenth-order dimension, centred about the nominal point, but of sizes $0.614,0.210$ and 0.288 respectively.

When uncertainties are not included in the model, the analysed system has the largest hypercube with a robust stability margin of 0.614 , as depicted in Table XIV. Although, the results may seem to be less conservative, they can not be guaranteed to be reliable, as the nominal parameters are rough estimates. With model uncertainties incorporated in the analysis, the robust stability margin is 0.210 and 0.288 for cases 4.2 and 4.3 respectively, representing smaller hypercubes with respect to case 4.1. Although, the results for cases 4.2 and 4.3 seem to be conservative in comparison to case 4.1 , they are more reliable. This is because the analyses take into account uncertainties of the system model, and therefore include worst case scenarios.

Further, in case 4.2 , the parametric space within which the system under study is robustly stable is smaller than in case 4.3 , as given by the aforementioned hypercubes. These findings indicate that the larger the uncertainty range, the tighter is the resulting robust stability margin, and the smaller the hypercube. While the size of their hypercubes, which represent the parametric space within which the systems are robustly stable, may differ, the results are reliable in both the cases since they include model uncertainties. It is to be pointed out that the reliability of the results is still dependent on the validity of the defined bounds of the uncertain system parameters.

Of note is that incorporating model uncertainties is computationally more expensive. This is evident since the size of the uncertainty matrix increases with the number of uncertain parameters. However, it is to be noted that the computation time is also dependent on the defined frequency grid. For very large uncertainty matrices, the computation time can be kept reasonably low by initially using a frequency grid of low density over a wide range of frequencies so as to obtain an estimated critical frequency value. $\mu$ analysis is then performed by narrowing the frequency range around the estimated critical frequency but keeping the initial number of frequency points. The analysis is eventually performed around the peak of the $\mu$ chart; this process may be repeated with higher number of frequency points over the very narrow frequency range until the $\mu$ values returned by the analyses remain consistent. For the case studies in this section, the $\mu$ results and computation times are given in Table $\mathrm{XV}$, based on a frequency grid of 25 points between 707 and $708 \mathrm{~Hz}$ for case 4.1, 708 and $709 \mathrm{~Hz}$ for case 4.2, 709 and $710 \mathrm{~Hz}$ for case 4.3. The analyses were performed on a Intel Core i7-3820 3.6 GHz processor with 32GB RAM.

TABLE XV: Cases 4.1, 4.2, 4.3 - System with load and model uncertainties, $\mu$ analysis results and computation time

\begin{tabular}{cccccc}
\hline $\begin{array}{c}\text { Case } \\
\text { study }\end{array}$ & $\begin{array}{c}\text { Model } \\
\text { uncertainties }\end{array}$ & $\begin{array}{c}\text { Robust } \\
\text { stability } \\
\text { margin }(1 / \mu)\end{array}$ & $\begin{array}{c}\text { Critical } \\
\text { load }\end{array}$ & $\begin{array}{c}\text { Size } \\
\Delta(j w)\end{array}$ & $\begin{array}{c}\text { Computation } \\
\text { time }\end{array}$ \\
\hline & & & & & \\
4.1 & No & 0.614 & $1.73 \Omega$ & $37 \times 37$ & $9 \mathrm{~s}$ \\
4.2 & Yes & 0.210 & $2.24 \Omega$ & $351 \times 351$ & $3.4 \mathrm{~h}$ \\
4.3 & Yes & 0.288 & $2.14 \Omega$ & $351 \times 351$ & $3.6 \mathrm{~h}$
\end{tabular}

The section of the work has important implications. Often times, the design engineer does not have sufficient information as to the exact values of the system components. Yet, the parameters may be estimated within some reasonable bounds. With the $\mu$ approach, the designer is offered the flexibility of determining the best trade-off between accuracy and practicality, by choosing the levels of details that are incorporated into modelling, depending on available information. The same system model is employed. Only the considered uncertain elements are defined differently.

\section{Summary OF RESUlTS}

In practice, power electronic systems are subject to variations in load, line resistance and operating temperature while their nominal system model is generally bound to contain parametric model uncertainties. This work has applied the $\mu$ tool to gauge the impact of the aforementioned uncertainties on the robust stability margin of the dc/dc buck converter system. The results are depicted in Table XVI. In the case study 1.1, $\mu$ analysis has determined that the example system becomes 
unstable when the output power is increased to $16 \mathrm{~W}$. It has been found in case study 2.1 that if the uncertain parameters $R$ and $R_{i n}$ are kept within $80.3 \%$ of their respective nominal values, the system under study can be ensured to be stable for an output power of up to $17.3 \mathrm{~W}$. For the case 2.1 , the robust stability margin increases as the source resistance $R_{i n}$, set in the range of [150 $\mathrm{m} \Omega, 450 \mathrm{~m} \Omega$ ], provides more damping to the resonant $L C$ input filter with respect to case 1.1, when $R_{\text {in }}$ is set at a constant value of $160 \mathrm{~m} \Omega$. The $\mu$ predictions for case studies 1.1 and 2.1 are supported by experimental results. Further, the robust stability margin of the buck converter has been found to be $74.5 \%$ when uncertainties in temperature are not included in case study 1.2 , as compared to $50.5 \%$ when uncertainties in temperature are included as shown in case study 3.1. The findings emphasise the necessity of incorporating operating conditions uncertainties for more reliable stability analysis of a system. The study has also demonstrated how the $\mu$ tool can be employed to account for model uncertainties, including certain classes of nonlinearities in the system model, such as in duty cycle $D$ in this work. $\mu$ analysis has predicted the critical output power of the considered buck converter to be $15.0 \mathrm{~W}$, when model uncertainties are neglected in case study 4.1. On the other hand, the critical output power has been determined as $11.6 \mathrm{~W}$ in case study 4.2 , when uncertainties are included, while its value increased to $12.2 \mathrm{~W}$, when the given uncertainties are defined within a relatively narrower range in case study 4.3. As discussed in section II, although, $\underline{\mu}$ is not guaranteed to be equal to $\mu$, it is generally close to $\mu$. For the cases investigated in this work, as the $\mu$ based predictions match closely the results from experiment, time domain simulations and eigenplots, it can be inferred that the lower bound $\mu$ is a good estimate of $\mu$.

\section{COnclusion}

This work has demonstrated practical approaches to applying the $\mu$ method in the assessment of robust stability of PE systems with single and multiple uncertainties, based on the widely employed dc/dc buck converter system. Further, it has shown the necessity of incorporating uncertainties in stability analysis. The $\mu$ tool has been used to determine the bounds within which the uncertain load must lie in order to guarantee the stability of the system under study. The analysis has been extended to include line resistance uncertainty. The $\mu$ predictions of the critical destabilising resistive load of the system under study, with and without line resistance uncertainty, have been validated in experiment. It has been shown how accounting for variation in operating temperature, which has an effect on multiple resistive system components, can increase the reliability of stability assessment. Further, the practical approach of including uncertainties, such as in parasitics and nonlinearity in duty cycle, in the nominal system model has been demonstrated. Although the robust stability margin has been found to be tighter when uncertainties are defined within wider ranges or with less accuracy, the work has shown that the results can be employed reliably as worse case scenarios are accounted for, which is particularly important for safety critical applications. In addition, the methodology for applying the $\mu$ tool as well as the interpretation of the $\mu$ results have been presented in a generalised and clear manner, which allows it to be extended to wider applications, and to include yet further sources of uncertainties.

\section{ACKNOWLEDGMENT}

This project has been conducted in the frame of the Clean Sky 2 Joint Undertaking under the European Union's Horizon 2020 research and innovation programme under grant agreement $N^{o}$ CS2-SYS-GAM-2014-2015-01.

\section{REFERENCES}

[1] I. E. Agency. (2009) Transport energy and $\mathrm{CO}_{2}$ : Moving towards sustainability. [Online]. Available: https://www.iea.org/publications/ freepublications/publication/transport2009.pdf

[2] H. Zhang, C. Saudemont, B. Robyns, and M. Petit, "Comparison of technical features between a more electric aircraft and a hybrid electric vehicle," in 2008 IEEE Vehicle Power and Propulsion Conference, Sept 2008, pp. 1-6.

[3] R. D. Middlebrook, "Input filter considerations in design and application of switching regulators," IAS Record, 1976, pp. 366-382, 1976.

[4] A. B. Jusoh, "The instability effect of constant power loads," in Power and Energy Conference, 2004. PECon 2004. Proceedings. National. IEEE, 2004, pp. 175-179.

[5] A. Emadi, A. Khaligh, C. H. Rivetta, and G. A. Williamson, "Constant power loads and negative impedance instability in automotive systems: definition, modeling, stability, and control of power electronic converters and motor drives," IEEE Transactions on Vehicular Technology, vol. 55, no. 4, pp. 1112-1125, July 2006.

[6] A. M. Rahimi and A. Emadi, "An analytical investigation of dc/dc power electronic converters with constant power loads in vehicular power systems," Vehicular Technology, IEEE Transactions on, vol. 58, no. 6, pp. 2689-2702, 2009.

[7] S. Rosado, R. Burgos, F. Wang, and D. Boroyevich, "Large-signal stability analysis in power systems with a synchronous generator connected to a large motor drive," in 2007 IEEE Electric Ship Technologies Symposium, May 2007, pp. 42-47.

[8] A. Riccobono and E. Santi, "Comprehensive review of stability criteria for DC power distribution systems," Industry Applications, IEEE Transactions on, vol. 50, no. 5, pp. 3525-3535, 2014

[9] G. F. Franklin, J. D. Powell, A. Emami-Naeini, and J. D. Powell, Feedback control of dynamic systems. Addison-Wesley Reading, 1994, vol. 2 .

[10] R. C. Dorf and R. H. Bishop, Modern control systems. Pearson (Addison-Wesley), 1998.

[11] K. Areerak, "Modelling and stability analysis of aircraft power systems," Ph.D. dissertation, The Department of Electrical and Electronic Engineering, University of Nottingham, 2009.

[12] W. E. Sollecito and D. A. Swann, "Computer evaluation of hightemperature aircraft a-c electrical system designs," Transactions of the American Institute of Electrical Engineers, Part II: Applications and Industry, vol. 78, no. 6, pp. 434-444, Jan 1960.

[13] J. Doyle, "Analysis of feedback systems with structured uncertainties," in IEE Proceedings D (Control Theory and Applications), vol. 129, no. 6. IET, 1982, pp. 242-250.

[14] K. Zhou, J. Doyle, and K. Glover, Robust and Optimal Control. Prentice Hall, 1996.

[15] M. Davari and Y. A. R. I. Mohamed, "Robust multi-objective control of vsc-based dc-voltage power port in hybrid ac/dc multi-terminal microgrids," IEEE Transactions on Smart Grid, vol. 4, no. 3, pp. 1597-1612, Sept 2013

[16] M. Davari and Y. A. R. I. Mohamed, "Dynamics and robust control of a grid-connected vsc in multiterminal dc grids considering the instantaneous power of dc- and ac-side filters and dc grid uncertainty," IEEE Transactions on Power Electronics, vol. 31, no. 3, pp. 1942-1958, March 2016.

[17] S. Sudhoff and O. Wasynczuk, "Analysis and average-value modeling of line-commutated converter-synchronous machine systems," Energy Conversion, IEEE Transactions on, vol. 8, no. 1, pp. 92-99, 1993.

[18] M. Kuhn, Y. Ji, and D. Schrder, "Stability studies of critical DC power system component for More Electric Aircraft using mu sensitivity," in Control \& Automation, 2007. MED'07. Mediterranean Conference on. IEEE, 2007, pp. 1-6. 
TABLE XVI: Summary of Results

\begin{tabular}{|c|c|c|c|c|c|c|c|}
\hline \multirow{2}{*}{$\begin{array}{l}\text { Case } \\
\text { study }\end{array}$} & \multirow{2}{*}{$\begin{array}{l}\text { Uncertain } \\
\text { parameters }\end{array}$} & \multirow{2}{*}{$\begin{array}{c}\text { Duty cycle } \\
D\end{array}$} & \multirow{2}{*}{$\begin{array}{c}\text { Nominal } \\
\text { values }\end{array}$} & \multirow{2}{*}{$\begin{array}{c}\text { Robust } \\
\text { stability } \\
\text { margin }\end{array}$} & \multirow[t]{2}{*}{$\underline{\mu}$} & \multicolumn{2}{|c|}{ Critical load } \\
\hline & & & & & & $R(\Omega)$ & power $(W)$ \\
\hline 1.1 & Load $R$ & $1^{s t}$ order approximation & Refined & 0.696 & 1.44 & $1.63 \Omega$ & $16.0 \mathrm{~W}$ \\
\hline 1.2 & Load $R$ & $0^{\text {th }}$ order approximation & Refined & 0.745 & 1.34 & $1.57 \Omega$ & $16.6 \mathrm{~W}$ \\
\hline 2.1 & Load $R$ and line resistance $R_{i n}$ & $1^{s t}$ order approximation & Refined & 0.803 & 1.24 & $1.50 \Omega$ & $17.3 \mathrm{~W}$ \\
\hline 3.1 & Load $R$ and temperature $T$ & $0^{t h}$ order approximation & Refined & 0.505 & 1.98 & $1.87 \Omega$ & $13.9 \mathrm{~W}$ \\
\hline 4.1 & Load $R$ and no model uncertainty & $0^{\text {th }}$ order approximation & Initial & 0.614 & 1.63 & $1.73 \Omega$ & $15.0 \mathrm{~W}$ \\
\hline 4.2 & Load $R$ and model uncertainties (wide range) & $D$ uncertain parameter & Initial & 0.210 & 4.76 & $2.24 \Omega$ & $11.6 \mathrm{~W}$ \\
\hline 4.3 & Load $R$ and model uncertainties (narrow range) & $D$ uncertain parameter & Initial & 0.288 & 3.47 & $2.14 \Omega$ & $12.2 \mathrm{~W}$ \\
\hline
\end{tabular}

[19] J. Elizondo, R. Y. Zhang, J. K. White, and J. L. Kirtley, "Robust small signal stability for microgrids under uncertainty," in Power Electronics for Distributed Generation Systems (PEDG), 2015 IEEE 6th International Symposium on. IEEE, 2015, pp. 1-8.

[20] P. M. Young, M. P. Newlin, and J. C. Doyle, " $\mu$ analysis with real parametric uncertainty," in Decision and Control, 1991., Proceedings of the 30th IEEE Conference on. IEEE, 1991, pp. 1251-1256.

[21] R. Tymerski, "Worst case stability analysis of switching regulators using the structured singular value," in Power Electronics Specialists Conference, PESC '94 Record., 25th Annual IEEE, Jun 1994, pp. 281288 vol.1.

[22] M. Green and D. J. Limebeer, Linear robust control. Courier Corporation, 2012.

[23] S. Skogestad and I. Postlethwaite, Multivariable Feedback Control: Analysis and Design. Wiley, 2005.

[24] A. Packard and J. Doyle, "The complex structured singular value," Automatica, vol. 29, no. 1, pp. 71-109, 1993.

[25] G. Ferreres, A practical approach to robustness analysis with aeronautical applications. Springer Science \& Business Media, 1999.

[26] A. Varga, G. Looye, D. Moormann, and G. Gräbel, "Automated generation of LFT-based parametric uncertainty descriptions from generic aircraft models," Mathematical and Computer Modelling of Dynamical Systems, vol. 4, no. 4, pp. 249-274, 1998.

[27] A. Fabrizi, C. Roos, and J.-M. Biannic, "A detailed comparative analysis of $\mu$ lower bound algorithms," in European Control Conference 2014, 2014.

[28] G. Balas, R. Chiang, A. Packard, and M. Safonov. (2005) Robust control toolbox 3 users guide.

[29] D. Piga, "Computation of the structured singular value via moment lmi relaxations," IEEE Transactions on Automatic Control, vol. 61, no. 2, pp. 520-525, Feb 2016.

[30] X. Chen and J. T. Wen, "Model reduction of multidimensional positive real systems," in Proceedings of 1994 33rd IEEE Conference on Decision and Control, Dec 1994, pp. 3758-3763.

[31] M. Ferber, A. Korniienko, G. Scorletti, C. Vollaire, F. Morel, and L. Krhenbhl, "Systematic lft derivation of uncertain electrical circuits for the worst-case tolerance analysis," IEEE Transactions on Electromagnetic Compatibility, vol. 57, no. 5, pp. 937-946, Oct 2015.

[32] J. C. Doyle, B. A. Francis, and A. Tannenbaum, Feedback control theory. Macmillan Publishing Company New York, 1992, vol. 1.

[33] R. Castellanos, C. Juarez, J. Hernandez, and A. Messina, "Robustness analysis of large power systems with parametric uncertainties," in Power Engineering Society General Meeting, 2006. IEEE. IEEE, pp. 1-8.

[34] M. Djukanovic, M. Khammash, and V. Vittal, "Application of structured singular value theory for robust stability and control analysis in multimachine power systems. II. Numerical simulations and results," IEEE Transactions on Power Systems, vol. 13, no. 4, pp. 1317-1322, Nov 1998.

[35] R. Castellanos, A. Messina, and H. Sarmiento, "Robust stability analysis of large power systems using the structured singular value theory," International Journal of Electrical Power \& Energy Systems, vol. 27, no. 5, pp. 389-397, 2005.

[36] M. Djukanovic, M. Khammash, and V. Vittal, "Application of the structured singular value theory for robust stability and control analysis in multimachine power systems. I. Framework development," IEEE Transactions on Power Systems, vol. 13, no. 4, pp. 1311-1316, Nov 1998.
[37] S. Chen and O. P. Malik, "Power system stabilizer design using $\mu$ synthesis," IEEE Transactions on Energy Conversion, vol. 10, no. 1, pp. 175-181, Mar 1995.

[38] A. Haddadi, B. Boulet, A. Yazdani, and G. Joós, "A $\mu$-based approach to small-signal stability analysis of an interconnected distributed energy resource unit and load," IEEE Transactions on Power Delivery, vol. 30, no. 4, pp. 1715-1726, 2015.

[39] D.-W. Gu, Robust control design with MATLAB®. Springer Science \& Business Media, 2005, vol. 1.

[40] S. Buso, "Design of a robust voltage controller for a buck-boost converter using $\mu$ synthesis," IEEE Transactions on Control Systems Technology, vol. 7, no. 2, pp. 222-229, Mar 1999.

[41] C. Zhang, J. Wang, S. Li, B. Wu, and C. Qian, "Robust control for pwmbased DC-DC buck power converters with uncertainty via sampled-data output feedback," IEEE Transactions on Power Electronics, vol. 30, no. 1, pp. 504-515, Jan 2015.

[42] J. A. Solsona, S. G. Jorge, and C. A. Busada, "Nonlinear control of a buck converter which feeds a constant power load," IEEE Transactions on Power Electronics, vol. 30, no. 12, pp. 7193-7201, Dec 2015.

[43] M. Fard and M. Aldeen, "Robust control design of a dc micro grid comprising photovoltaic and battery systems," in 2016 IEEE PES AsiaPacific Power and Energy Engineering Conference (APPEEC), Oct 2016, pp. 329-336.

[44] S. Sumsurooah, M. Odavic, S. Bozhko, and D. Boroyevich, "Stability and robustness analysis of a $\mathrm{dc} / \mathrm{dc}$ power conversion system under operating conditions uncertainties," in Industrial Electronics Society, IECON 2015 - 41st Annual Conference of the IEEE, Nov 2015, pp. $003110-003115$.

[45] S. Sumsurooah, M. Odavic, and S. Bozhko, "A modeling methodology for robust stability analysis of nonlinear electrical power systems under parameter uncertainties," IEEE Transactions on Industry Applications, vol. 52, no. 5, pp. 4416-4425, Sept 2016.

[46] S. Sumsurooah, M. Odavic, and S. Bozhko, "Development of lft-based models for robust stability analysis of a generic electrical power system over all operating conditions," in 2015 International Conference on Electrical Systems for Aircraft, Railway, Ship Propulsion and Road Vehicles (ESARS), March 2015, pp. 1-6.

[47] S. Sumsurooah, M. Odavic, and S. Bozhko, " $\mu$ approach to robust stability domains in the space of parametric uncertainties for a power system with ideal CPL," IEEE Transactions on Power Electronics, vol. PP, no. 99, pp. 1-1, 2017.

[48] S. Sumsurooah, M. Odavic, and D. Boroyevich, "Modelling and robust stability analysis of uncertain systems," in 2013 Grand Challenges on Modeling and Simulation Conference. Society for Modeling \& Simulation International, 2013.

[49] G. J. Balas, J. C. Doyle, K. Glover, A. Packard, and R. Smith. (2001) $\mu$-Analysis and Synthesis Toolbox: For Use with MATLAB.

[50] R. W. Erickson and D. Maksimovic, Fundamentals of power electronics. Springer Science \& Business Media, 2001.

[51] H. J. Zhang, "Modeling and loop compensation design of switching mode power supplies," Linear Technology, Appl. Note 149, 2015.

[52] A. A. Elbaset, "Small-signal matlab/simulink model of dc-dc buck converter using state-space averaging method," in 17th International Middle-East Power System Conference (MEPCON'15) Mansoura University, Egypt, December 15-17, 2015, pp. 1-8.

[53] D. Mattingly, "Designing stable compensation networks for single phase voltage mode buck regulators," Intersil, Tech. Rep. TB417.1, Dec. 2003. 
[54] D. Meeks, "Loop stability analysis of voltage mode buck regulator with different output capacitor types-continuous and discontinuous modes," Texas Instruments, App. Note SLVA301, 2008. 